\title{
Rare variant analyses across multiethnic cohorts identify novel genes for refractive error
}

\author{
Anthony Musolf \\ National Institutes of Health \\ Annechien Haarman \\ Erasmus Medical Center https://orcid.org/0000-0002-1452-5700 \\ Robert Luben \\ University of Cambridge School of Clinical Medicine \\ Jue-Sheng Ong \\ QIMR Berghofer Medical Research Institute https://orcid.org/0000-0002-6062-710X \\ Karina Patasova \\ King's College London \\ Rolando Hernandez Trapero \\ University of Edinburgh \\ Joseph Marsh \\ University of Edinburgh \\ Ishika Jain \\ National Institutes of Health \\ Riya Jain \\ National Institutes of Health \\ Paul Zhiping Wang \\ University of Pennsylvania \\ Deyana Lewis \\ National Institutes of Health \\ Milly Tedja \\ https://orcid.org/0000-0003-0356-9684
}

\section{Adriana Iglesias}

Erasmus University Medical Center https://orcid.org/0000-0001-5532-764X

\section{Hengtong Li}

National University of Singapore

\section{Cameron Cowan}

Institute for Molecular and Clinical Ophthalmology

CREAM Consortium (Representative Caroline Klaver)

Erasmus Medical Center

\section{Ginevra Biino}

National Research Council of Italy

\section{Alison Klein}

Department of Oncology, Sidney Kimmel Comprehensive Cancer Center, Johns Hopkins School of Medicine https://orcid.org/0000-0003-2737-8399

\section{Priya Duggal}


Johns Hopkins University https://orcid.org/0000-0001-5809-2081

\section{David Mackey}

University of Western Australia

\section{Caroline Hayward}

University of Edinburgh

\section{Toomas Haller}

Estonian Genome Center, Institute of Genomics, University of Tartu https://orcid.org/0000-0002-5069-6523

\section{Andres Metspalu}

The Estonian Genome Center, University of Tartu https://orcid.org/0000-0002-3718-796X

\section{Juho Wedenoja}

University of Helsinki and Helsinki University Hospital

\section{Olavi Pärssinen}

University of Jyväskylä

\section{Ching-Yu Cheng}

Singapore Eye Research Institute, Singapore National Eye Centre; Ophthalmology and Visual Sciences Academic Clinical Program (Eye ACP), Duke-NUS Medical School, Singapore https://orcid.org/0000-0003-0655-885X

\section{Seang Mei Saw}

Yong Loo Lin School of Medicine, National University of Singapore

\section{Dwight Stambolian}

University of Pennsylvania

\section{Pirro Hysi}

King's College London https://orcid.org/0000-0001-5752-2510

\section{Anthony Khawaja}

University College London https://orcid.org/0000-0001-6802-8585

\section{Veronique Vitart}

University of Edinburgh https://orcid.org/0000-0002-4991-3797

\section{Christopher Hammond}

King's College London https://orcid.org/0000-0002-3227-2620

\section{Cornelia van Duijn}

University of Oxford https://orcid.org/0000-0002-2374-9204

\section{Virginie Verhoeven}

Erasmus Medical Center https://orcid.org/0000-0001-7359-7862

\section{Caroline Klaver}

Erasmus Medical Center https://orcid.org/0000-0002-2355-5258

\section{Joan Bailey-Wilson ( $\nabla$ jebw@mail.nih.gov )}

National Human Genome Research Institute, NIH https://orcid.org/0000-0002-9153-2920

\section{Article}

Keywords: refractive error, genetic risk factors, rare variants

Posted Date: September 3rd, 2021

DOl: https://doi.org/10.21203/rs.3.rs-846265/v1 
License: (1) This work is licensed under a Creative Commons Attribution 4.0 International License. Read Full License

Version of Record: A version of this preprint was published at Communications Biology on January 3rd, 2023. See the published version at https://doi.org/10.1038/s42003-022-04323-7. 


\section{Abstract}

Refractive error is a complex eye condition caused by both genetic and environmental factors. Common genetic risk factors have been identified by genome-wide association studies (GWAS), but a great part of the refractive error heritability is still missing. Some of this heritability may be explained by rare variants (minor allele frequency [MAF] $\leq$ 0.01.). We performed multiple gene-based association tests for rare variants on exome array data from the Consortium for Refractive Error and Myopia (CREAM). The dataset consisted of over 27,000 total subjects from five cohorts of IndoEuropean and East Asian ethnicity. We identified 129 unique genes associated with refractive error, many of which were replicated in multiple cohorts. Our best novel candidates included the retina expressed $P D C D 6 I P$, the circadian rhythm gene $P E R 3$, and P4HTM, which affects eye morphology. Future work will include functional studies and validation.

\section{Introduction}

Refractive error has become a major worldwide health concern, with the prevalence of the disease, particularly myopia (nearsightedness), becoming more frequent in both the United States ${ }^{1}$ and Europe ${ }^{2}$ and reaching epidemic proportions in parts of East Asia ${ }^{3,4}$. Refractive error is caused when the optics of the eye fail to project the focal point of light on the retina, causing a blurred image. Myopia is the refractive error mostly resulting from eye elongation, which can lead to serious ocular complications like myopic macular degeneration, glaucoma and retinal detachment ${ }^{5-8}$, and is the second most common cause of blindness ${ }^{9-11}$.

Refractive error is a highly complex trait that is known to have both an environmental and genetic etiology. Established environmental factors include prolonged near work, education, and outdoor exposure ${ }^{12}$. Genome-wide association studies (GWAS) and genetic linkage studies have identified multiple associated variants for refractive error ${ }^{13-18}$. The Consortium for Refractive Error and Myopia (CREAM) has reported numerous risk variants using large-scale, multiethnic datasets $^{19-22}$, explaining approximately $18 \%$ of phenotypic variance ${ }^{22}$.

Despite estimates that $50-80 \%$ of refractive error variance is determined by genetic factors ${ }^{23-26}$, much of the refractive error heritability remains unaccounted for ${ }^{19,21}$. Since GWAS are particularly designed to identify common variants, some of the missing heritability may lie with rare variants (minor allele frequency $[M A F] \leq 0.01$ ), which may be highly penetrant and exert a large effect on the phenotype ${ }^{27}$. Gene-based association tests, such as burden-style tests ${ }^{28,29}$, offer increased power to find rare variants not identified by GWAS.

This study performs the first large-scale rare variant analysis on refractive error using multiethnic cohorts from CREAM. We used an initial discovery dataset consisting of over 13,000 Indo-Europeans and four replication datasets consisting of European ancestry Americans, European ancestry Australians, European ancestry Britons, and East Asian ancestry Singaporeans. Gene-based tests were performed on each of the five cohorts and meta-analysis was performed subsequently. Pathway analysis was conducted on genome-wide significant genes and genes were prioritized based on annotation and biologic relevance to the trait.

\section{Methods}

\section{Cohort Details, Genotyping and Joint Recalling of Exome Array Data}

Fourteen population-based CREAM cohorts that had exome chip genotypes on individuals with refractive error were used in this study. These 14 cohorts were: Singapore Chinese Eye Study (SCES), Singapore Malay Eye Study (SiMES), Singapore Indian Eye Study (SINDI), Age Related Eye Study (AREDS), Rotterdam Study I (RSI), Erasmus Rucphen Family 
(ERF), Raine Eye Health Study (REHS), Beaver Dam Eye Study (BDES), Estonian Genome Center for the University of Tartu (EGCUT), Finnish Twin Study on Aging (FITSA), Ogliastra, Croatia-Korcula, TwinsUK, and EPIC-Norfolk. Each individual cohort is described in further detail in the Supplementary Methods. All studies were performed in accordance with the Declaration of Helsinki and approved by the institutional review boards of the participating institutions. All participants provided written informed consent.

Thirteen cohorts had been genotyped on the Illumina HumanExome-12 v 1.0 or v 1.1, or the Illumina HumanCoreExome12 v1.0; EPIC-Norfolk was genotyped on Affymetrix UK BioBank Axiom Array. The 13 cohorts on the Illumina arrays were jointly recalled to obtain a larger sample size of rare variants (here defined as variants with a MAF $\leq 0.01$ ), as recalling genotypes simultaneously across all samples increases the ability to call rare variants with more discrete distinction between allele calls and sensitivity for low-frequency (high-intensity) loci. All data were recalled using GenomeStudio® v2011.1 (Illumina Inc., San Diego, CA, USA) per microarray platform and PLINK ${ }^{30}$.

\section{Combination of Cohorts for Mega-analysis}

To increase power on rare variants, we sought to combine as many cohorts as possible into a mega-analysis. We thus performed principal components analysis (PCA) on all our cohorts after pruning the datasets for linkage disequilibrium using the pcair, part of the R package GENESIS. Pcair is designed to perform PCA in samples with cryptic relatedness and provides accurate ancestry inference that is not confounded by family structure ${ }^{31}$. For reference, we included individuals from all 11 HapMap reference panels in the PCA.

PCA showed two major groupings based on known ethnicity. The first consisted of the Han Chinese SCES and Malaysian SiMES cohorts, which were combined into the East Asian combined cohort (EACC). The second dataset consisted of the eight European cohorts (RSI, Croatia-Korcula, FITSA, EGCUT, TwinsUK, ERF, AREDS, and Ogliastra) and the one Indian cohort (SINDI). These cohorts were combined into the Indo-European combined cohort (IECC).

Analysis was performed on five discrete cohorts - IECC, EACC, EPIC-Norfolk, BDES, and REHS. The IECC analysis was performed in the Netherlands, while the EACC was performed in the United States as well as in the Netherlands. The BDES, EPIC-Norfolk, and REHS analyses were performed in their countries of origin (the United States, the United Kingdom, and Australia, respectively) as was legally required; these studies served on a per study basis as replication cohorts. A breakdown of all cohorts and the combined cohort with which they are grouped is provided in Supplementary Table 1.

\section{Quality Control}

For the combined cohorts, all raw cohort data were merged into a single file. All five cohorts then underwent identical quality control using PLINK ${ }^{30}$. Any individual not genotyped at $99 \%$ of all variants was removed and any variant not genotyped at $99 \%$ or not in HWE was also removed. We also checked for batch effects and calculated the identity-bydescent (IBD) value of all individuals in the cohort, removing duplicates and twins. Many of the datasets exhibited cryptic relatedness amongst subjects (especially the Ogliastra study, which collected on the Italian island of Sardinia). Related individuals were not removed from the cohorts, as our analysis methods corrected for relatedness. After QC, IECC had 13,037 individuals with 150,619 variants, EACC had 4,867 individuals with 98,750 variants, BDES had 1,740 individuals with 105,671 variants, REHS had 1,020 individuals with 92,313 variants, and EPIC-NORFOLK had 6,282 individuals with 637,160 variants.

\section{Refractive Error Phenotype}

Refractive error was defined as the quantitative phenotype spherical equivalent (SER), measured in diopters (D). Refractive error measurements in both eyes were taken from all participants and SER was calculated by adding the 
spherical refractive error + half the cylindrical refractive error in each eye, then taking the mean of both eyes. Individuals who had undergone procedures that could alter refraction, e.g., cataract surgery, laser refractive error procedures, retinal detachment surgery, and other ophthalmic conditions that may influence refraction were excluded from these analyses. The average spherical equivalents and standard deviations of each cohort are provided in Supplementary Table 1.

\section{Gene-based Analysis using EMMAX-VT and EMMAX-CMC}

Gene-based analysis was performed using a gene-based version of EMMAX. ${ }^{32,33}$ EMMAX uses a kinship matrix to correct for population stratification and cryptic relatedness, which are present in these cohorts. EMMAX has been modified to perform gene-based burden-style tests, including the variable threshold (VT) ${ }^{29}$ and the combined multivariate and collapsing $(\mathrm{CMC})^{28}$ methods through the software EPACTS (https://genome.sph.umich.edu/wiki/EPACTS), which we will term EMMAX-VT and EMMAX-CMC, respectively. ${ }^{34}$. The major difference between these two tests is the EMMAX-CMC assumes that all variants within the gene-based marker act in the same direction upon the value of the quantitative trait, SER, while EMMAX-VT allows for them to act in opposite directions.

We analyzed all five cohorts with EMMAX-VT and EMMAX-CMC using a maximum MAF $=0.01$. We only included variants that were in an exon of a gene (as defined by RefSeq), including both nonsynonymous and synonymous variants. Genes with a minor allele count (MAC) of less than three for the cohort were dropped from the analysis.

Initial analyses were performed without any covariates. We performed two follow-up analyses using age, sex, and education level (low, intermediate, and high). One covariate analysis included all three covariates, while the second used age and sex only (education level removed). We note that the inclusion of covariates resulted in no significance difference between significant genes; for brevity we only discuss the results without covariates. In addition, the Ogliastra cohort did not have data on age and education resulting in approximately 3,000 individuals being removed from the IECC covariate analyses.

\section{Gene-based Analysis using ACAT}

The Aggregated Cauchy Association Test (ACAT) ${ }^{35}$ is a novel method that allows individual p-values to be combined into a gene-based p-value that is particularly useful for rare variants. To take advantage of this method, we analyzed all variants with a MAF $\leq 0.01$ (with a minimum allele count of 3 ) using the original, single variant-based version of EMMAX. ${ }^{32,33}$ We then combined the EMMAX p-values for each gene using the ACAT package implemented through R. Only nonsynonymous and synonymous exonic variants were included in the analysis.

\section{Meta-Analysis and Replication}

The burden-style tests that created a single p-value for a gene precluded the use of popular meta-analysis programs such as METAL, which require the input of reference and alternative alleles. Instead the gene-based $p$-values from the EMMAX-VT, EMMAX-CMC and ACAT were combined across studies using the classic method described by Fisher ${ }^{36}$. Fisher's method was implemented through the R package metap ${ }^{37}$. We defined genome-wide significant as $1 \times 10^{-5}$, based on the standard for gene-based studies. Replication was defined as a having a $\mathrm{P} \leq 0.05$ in one cohort after being found to be genome-wide significant in one of the other four cohorts.

We performed two separate meta-analyses. The first combined all five cohorts (IECC, EACC, BDES, EPIC-Norfolk, and REHS), which will be referred to as the multiethnic meta-analysis. The second combined the four ethnically IndoEuropean cohorts (IECC, BDES, REHS, and EPIC-Norfolk), which will be referred to as the Indo-European meta-analysis. The Indo-European meta-analysis was designed to identify any genes that might be significant in Indo-Europeanderived individuals but not significant in East Asians; thus, we also report the East Asian analyses p-values. 
To investigate whether signals identified by the rare variant analysis were being partially driven by common variants, we calculated polygenic risk scores (PRS) for all cohorts using common variants identified in previous GWAS ${ }^{22}$. PRS were calculated for each subject using PLINK (Supplementary Table 2). All rare variant analyses were then repeated using the PRS values for each subject as a covariate. We compared the explained variance $\left(R^{2}\right)$ of our top individual genes between the analysis with and without including PRS (Supplementary Table 3-4).

Independent replication of the genome-wide significant genes was performed in the UK Biobank (UKBB) via extraction of all rare variants comprising the genome-wide significant genes and repeating the same analyses.

\section{Pathway and Expression Analysis}

All genome-wide significant genes in the four meta-analyses and the EACC analyses were analyzed using Ingenuity Pathway Analysis (IPA) (QIAGEN Inc., https://digitalinsights.qiagen.com/products-overview/discovery-insightsportfolio/analysis-and-visualization/qiagen-ipa/) ${ }^{38}$. We performed various analyses through IPA, including canonical pathway analysis (identifying which genes are in known pathways), upstream regulator analysis (which identifies genes, RNAs, and proteins that regulate the genes in the dataset), and causal network analysis (which expands the pathway analysis to include the upstream regulators in the pathway analysis). IPA also identified disease phenotypes, cellular/molecular functions, and physiological networks associated with the genes in the dataset. Additional pathway and expression analysis were also performed with Functional Mapping and Annotation of GWAS 39,40 (FUMA), which provided tissue-enrichment information from GTEx and gene-group information from MsigDB. We repeated the IPA and FUMA analyses for our top prioritized genes from the schema proposed below.

\section{Gene Prioritization based on Biological Function}

To prioritize genes according to biological background, we evaluated genes following a modified schedule proposed by Fritsche et al. ${ }^{41}$ and further adapted by Tedja et al. ${ }^{21}$ Genes were ranked based on points equally assigned for the presence of replication, expression and biological plausibility. Evidence for ocular expression was based on single-cell expression data from adult human retina and developed organoids ${ }^{42}$. Biological plausibility was based on the presence of an ocular phenotype in OMIM and/or DisGeNET ${ }^{43}$ as well as an ocular phenotype in a knock-out mouse model of this gene (Mouse Genome Informatics and International Mouse Phenotyping Consortium databases). The prioritization score ranged from zero to seven. In addition, we performed a look-up of the top-genes to screen for drugs that had these genes as target using SuperTarget ${ }^{44}$, PharmGkb, ${ }^{45}$ STITCH v5.0 ${ }^{46}$ and DrugBank v5.0. ${ }^{47}$

\section{Variant Annotation for Potential Causal Variants}

We performed annotation to identify potential causal variants within the significant genes. Therefore, we annotated all exonic variants from genome-wide significant genes using WANNOVAR ${ }^{48-50}$, which collates functional predictions from popular prediction algorithms like SIFT ${ }^{51}$, PolyPhen $2^{52}$, MutationTaster ${ }^{53}$, CADD $^{54}$, and FATHMM ${ }^{55}$. We initially looked at the top-ranked genes in the prioritization approach described above, giving preference to variants that appeared to either be driving the gene-based association analysis or variants that the five annotation algorithms agreed upon as being damaging. We further expanded this approach to all significant genes identified in the meta-analyses.

\section{Structural Analysis of Variants}

We also performed structural analysis of all coding variants within our top prioritized genes, as well as all mutations predicted to be deleterious in all genome-wide significant genes. Crystal structures were obtained from the Protein Data Bank $^{56}$; when crystal structures were not available, homology models were used for visualization and energy calculations. We used both FoldX RepairPDB and Position Scan ${ }^{57}$ to predict differences in free energy between the wildtype and mutant proteins $(\Delta \Delta \mathrm{G}$, measured in $\mathrm{kcal} / \mathrm{mol})$. ChimeraX ${ }^{58}$ was used to visualize affected proteins. We 
also incorporated prior information from publicly available databases (OMIM, PFam, ClinVar, gnomAD, UniProt, RCSB PDB) and predicted functional effects (Missense $3 D^{59}$ ).

\section{Results}

\section{Overview of all Analyses}

Across the three (i.e., VT, CMC and ACAT) multiethnic meta-analyses, the three Indo-European meta-analyses and the three EACC analyses, we identified a total of 129 unique genes that were significantly associated with the refractive error phenotype (Supplementary Tables 3-5). We found no statistically significant difference in p-value or the number of unique genome-wide significant genes when adding the PRS as covariates.

\section{Multiethnic Meta-analyses}

Forty-three genome-wide significant genes were found using EMMAX-VT (Fig. 1A), 11 genome-wide significant genes using the EMMAX-CMC (Fig. 1B), and 28 genome-wide significant genes using ACAT (Fig. 1C).

Sixty-eight unique genes were identified across the three tests (Fig. 2). Four genes were significant across all three tests - GDF15 (19p13.11), PDCD6IP (3p22.3), RRM2 (2p25.1), and ST6GALNAC5 (1p31.1). GDF15 (19p13.11) was one of the top two significant genes in all three approaches (EMMAX-VT P $=5.12 \times 10^{-9}$, EMMAX-CMC $\mathrm{P}=1.12 \times 10^{-9}, A C A T P=$ $1.95 \times 10^{-9}$ ). GDF15, PDCD6IP, and RRM2 all replicated in at least one cohort; ST6GALNAC5 only appeared in IECC and thus could not be replicated.

Overall, 25 genes were replicated using the EMMAX-VT approach, 11 in the ACAT approach, and 4 in the EMMAX-CMC approach. Three genes - HCAR1, CCDC9, and NINJ2 - were replicated in more than one replication cohort, all in the EMMAX-VT approach. MRPS27 in EMMAX-VT (REHS and EPIC-Norfolk) and GDF15 in ACAT (IECC and REHS) had genome-wide significant p-values in two cohorts. The list of all genome-wide significant genes for each test can be found in Supplementary Tables 6-8, while the full results of all p-values can be found in Supplementary Tables 9-11.

\section{Indo-European Meta-analyses}

As it is possible that East Asians differ in genetic risk factor profile from Indo-Europeans, we performed meta-analyses on the four Indo-European ancestry cohorts. Forty-nine genes were genome-wide significant in the EMMAX-VT approach (Fig. 3A), 13 genes in the EMMAX-CMC approach (Fig. 3B), and 29 genes in the ACAT approach (Fig. 3C). Four genes overlapped between all three tests - GDF15, PIK3CA, RRM2, and ST6GALNAC5 (Fig. 4). The signal at PIK3CA was unique to the Indo-European meta-analysis. GDF15 and RRM2 were both replicated in one cohort, while PIK3CA and ST6GALNAC5 only appeared in IECC.

Overall, 24 genes were replicated in EMMAX-VT, 8 genes in ACAT, and 4 genes in EMMAX-CMC. NINJ2 in the EMMAX-VT and STON1 and SND1 in EMMAX-CMC were replicated in multiple cohorts. The list of all genome-wide significant genes for each test can be found in Supplementary Tables 12-14, while the full results of all p-values can be found in Supplementary Tables 15-17.

\section{EACC Analysis}

We also report the standalone results of EACC analysis. Thirty-one genome-wide significant genes were found in EACC using EMMAX-VT (Fig. 5A), 5 genome-wide significant genes using EMMAX-CMC (Fig. 5B), and 22 genome-wide significant genes using ACAT (Fig. 5C). GSTM5 (1p13.3) and WEE1 (11p15.4) overlapped in all three tests (Fig. 6). SERTAD3 (chromosome 19) and ZNF25 (chromosome 10) were genome-wide significant and only appeared in EACC, 
i.e., rare variants in these two genes did not exist in the other cohorts. 51 unique genome-wide significant genes were identified, 39 novel to the EACC analyses. The list of all genome-wide significant genes for each test can be found in Supplementary Tables 18-20.

\section{Cohort Unique Genes}

In addition to the two genes in the EACC EMMAX-VT analysis, there were 6 significantly associated genes that only had rare variants within a single cohort; no other rare variants existed in the other cohorts for these genes. EDN3 and CHMP1B in the IECC EMMAX-VT analysis and PRLH in the IECC ACAT analysis. KLF1 appeared only in the EPIC-Norfolk cohort, in both the EMMAX-VT and EMMAX-CMC analyses. The list of cohort unique genes appears in Supplementary Table 21.

\section{Independent Replication in UK Biobank}

We extracted the variants from the 129 significant unique genes and performed replication analyses in the UK Biobank. There were 7 genes with a $\mathrm{P}<0.05$ in EMMAX-CMC and 9 genes with a $\mathrm{P}<0.05$ in EMMAX-VT (Supplementary Table 22). P4HTM, CCDC170, and CPB1 were found in both analyses. STON1 was also replicated in the UK Biobank analyses; this gene had a significant meta-analysis p-value in the EMMAX-CMC analysis. Interestingly, the p-value in all cohorts was $<0.053$.

\section{Pathway and Expression Analysis on all Significant Genes}

We performed IPA pathway analysis on the 129 unique genes. While this did not result in any genome-wide significant canonical pathways, the upstream regulators analysis identified over 172 associated transcription factors. The two highest were the cytokine CSF2, which is known to regulate neuroglia after retinal injuries ${ }^{60}$, and the Transcription factor (TF) MEF2C, which is known to be expressed in the retina and controls photoreceptor gene expression ${ }^{61}$ (Supplementary Table 23). The fourth ranked p-value was the Raf kinases, which are known to be involved in retinal development ${ }^{62}$ and cell survival ${ }^{63}$; the fifth ranked $p$-value was $T B X 5$, which is expressed in the retina and involved in eye morphogenesis ${ }^{64,65}$. Causal network analysis identified 288 associated pathways (Supplementary Table 24), including the TRPC5 pathway, which regulates axonal outgrowth in developing ganglion cells ${ }^{66}$.

The top overall associated physiological system functions were organ morphology, organismal development and embryonic development, while the top molecular/cellular functions were cell cycle and cellular assembly/organization. Cancer and organismal injuries/abnormalities were the top overall associated phenotypes (Supplementary Table 25). Six genes were associated with ophthalmic phenotypes: CHST6, GCNT2, P4HTHM, USH2A, GRHL2, and MAPT.

FUMA analysis found that the top enriched tissues were heart, brain, muscle, and adipose tissue (Supplementary Fig. 1A). The top functional categories were cytoskeleton organization, cell cycle processes, mitotic nuclear division, and organelle organization (Supplementary Fig. 1B.

\section{Biological Plausibility and Prioritization of Genes}

Of the 129 genome-wide significant genes from the six meta-analyses, $27.9 \%$ (36/129) have a known expression in human ocular tissue. $51.2 \%(66 / 129)$ of these genes showed evidence for a human ocular phenotype.

Seven genes had a biological plausibility score higher than $3-P E R 3$ (internally replicated, expressed in ocular tissue and associated ocular phenotype, i.e., score of 5) and PDCD6IP, MAPT, CHST6, GRHL2, USH2A, and P4HTM (all with a score of 4). An additional 11 genes had a score of 3 - GDF15, RRM2, HSPH1, TPR, KRT81, SPHK1, GSTM5, THSD7A, 
$W E E 1$, and $B U B 1 B$ (Fig. 7). Detailed background for the prioritization of the genes can be found in Supplementary Tables 26A-F.

The highest overall biological plausibility score belonged to the circadian rhythm gene $P E R 3(1 \mathrm{p} 36)$. It was genomewide significant in both the all cohorts ACAT and Indo-European only meta-analyses $\left(P=1.08 \times 10^{-6}\right.$ and $1.15 \times 10^{-6}$, respectively); it was genome-wide significant in REHS and replicated in IECC. Circadian rhythm genes have been shown to be associated with refractive error ${ }^{22}$ and PER3 is located near the site of a known myopia locus (MYP14) at which the causal gene has not been identified ${ }^{67-69}$. PER3 was expressed in ON and OFF bipolar cells. Defects in this gene are associated with familial advanced sleep phase syndrome (OMIM 616882) and may contribute to other circadian phenotypes by altering the sensitivity to light $^{70}$. In defocus experiments in chicks using - 15D lenses, PER3 expression decreased by -1.26 fold in the retina ${ }^{71}$. Further chick defocusing experiments, showed that PER3 expression in the retina varies under altered visual conditions ${ }^{72}$.

Five genes had a score just below $P E R 3$, including the apoptosis gene PDCD6IP(3p22.3). This gene was found to be genome-wide significant in all-cohorts meta-analyses using all three tests $\left(P=1.07 \times 10^{-7}, 1.45 \times 10^{-7}\right.$, and $4.88 \times 10^{-6}$, respectively). Further $P D C D 6 I P$ had a $\mathrm{P}$ of $<0.006$ in both the EACC and IECC cohorts and did not appear in the other cohorts. It is particularly interesting because it has two low single variant p-values in both IECC and EACC ( 0.00556 and 0.00548 , respectively) and there are no rare variants in this gene in any of the other cohorts. PDCD6IP is expressed in ganglion cells of peripheral retina and plays a role in programmed cell death in uveal melanoma ${ }^{73}$ and may play a role in cornea lymphangiogenesis and vascular responses. ${ }^{74}$

MAPT (17q21.32) encodes tau proteins responsible for stabilizing microtubules; it was found to be genome-wide significant in the all cohorts EMMAX-VT analysis $\left(P=8.57 \times 10^{-7}\right)$. It was genome-wide significant in REHS and replicated in EPIC-Norfolk. Abnormal MAPT was present in human glaucoma patients with uncontrolled intraocular pressure ${ }^{75}$ Cowan et al. showed that MAPT was expressed in several cell types in both the peripheral and foveal human retina: horizontal cells, rod bipolar cells, ON and OFF bipolar cells GLY and GABA amacrine cells and ganglion cells ${ }^{42}$. A knock-out mouse model showed decreased total retina thickness.

CHST6 (16q23.1) was genome-wide significant in both the all cohorts and Indo-European only EMMAX-VT metaanalyses $\left(P=8.99 \times 10^{-7}\right.$ and $2.42 \times 10^{-7}$, respectively). The gene was genome-wide significant in IECC and replicated in BDES; it was also nearly replicated in EPIC-Norfolk. CHST6 plays a role in maintaining corneal transparency. Mutations in this gene may result in macular corneal dystrophy (OMIM 217800), which is characterized by bilateral, progressive corneal opacification and a reduction of corneal sensitivity. ${ }^{76}$ The mouse phenotype of a knock-out model corresponded to that of human, i.e. abnormal cornea morphology and decreased corneal (stroma) thickness. Since our reference expression database did not contain any corneal tissue, we couldn't score this category.

The transcription factor GRHL2 (8q22.3) was genome-wide significant in the all cohorts EMMAX-VT meta-analysis ( $\mathrm{P}=$ $\left.1.42 \times 10^{-6}\right)$. It was genome-wide significant in REHS and replicated in IECC. Mutations in GRHL2 may lead to posterior polymorphous corneal dystrophy ${ }^{77}$ (OMIM 618031), characterized by a variable phenotype ranging from an irregular posterior corneal surface with occasional opacities, corneal edema, reduced visual acuity, secondary glaucoma, and corectopia.

The transmembrane prolyl hydroxylase P4HTM (3p21.31) was only genome-wide significant in EACC using EMMAX-VT $\left(P=1.00 \times 10^{-7}\right)$. However, this gene was replicated independently in the UKBB analysis. P4HTM has been shown to be expressed in different ocular cells (including horizontal cells and bipolar cells). It is associated with HIDEA, a severe

Page $10 / 30$ 
autosomal recessive disorder that is characterized by multiple symptoms, including eye abnormalities ${ }^{78}$ (OMIM 618493) and knock-out mice models have shown abnormal eye morphology ${ }^{79}$.

The membrane gene USH2A (1q41) was genome-wide significant in the EACC ACAT analysis $\left(\mathrm{P}=7.55 \times 10^{-9}\right)$. It is well known to cause both Usher syndrome, which includes retinitis pigmentosa (RP) and mild to moderate hearing loss, as well as RP without hearing loss ${ }^{80}$. It is known to be expressed in the retina ${ }^{81}$.

\section{Pathway and Expression Analysis on Top Prioritized Genes}

We ran the IPA and FUMA analyses on the seven top prioritized genes. IPA did not identify any canonical pathways as significant; the only pathway shared across the genes was the 14-3-3-mediated signaling pathway (MAPT and PDCD6IP). The 14-3-3 proteins are a diverse group of signaling proteins.

Upstream regulator analysis found several transcription regulators of at least two genes include NKX2-1 (GRHL2 and MAPT), PSEN1 (MAPT and PER3), and SIRT1 (MAPT and PDCD6IP) (Supplementary Table 27). In the causal network analysis, the master regulator with the highest $\mathrm{p}$-value covering multiple genes was the cytokine macrophage migration inhibitory factor (MIF) (Supplementary Table 28), which covered five genes. Interestingly, MIF is an essential factor in the development of zebrafish eyes ${ }^{82}$ and has been found to be a potential regulator of diabetic retinopathy ${ }^{83}$. MIF inhibitors may also be protective to photoreceptors ${ }^{84}$. The top functional analysis for disease result was hereditary eye disease (Supplementary Table 29). FUMA showed the top tissue expression occurred in the small intestinal terminal ileum, skeletal muscle, and the brain cortex; the latter being probably the best proxy for eye tissue (Supplementary Fig. 2A). A heat map of the expression of the seven genes across all GTEx tissues is given in Supplementary Fig. 2B).

\section{Potential Causal Variants in the Prioritized Genes}

We used annotation from wANNOVAR to identify potential causal variants within the top genes identified by the prioritization method (Table 1). For the two prioritized genes that were significant in the ACAT analyses, we were able to look at single variant $\mathrm{p}$-values in addition to annotation to determine potential causal variants. There were three good candidate variants in PDCD6IP, which was genome-wide significant in IECC and replicated in EACC. rs199990824 appeared in the EACC only, was predicted to be damaging by SIFT and MutationTaster, and had a CADD score of 26. The minor allele of rs199990824 appeared in 37 carriers (all heterozygotes) with an average SER of -2.04 D (SD = 3.29) compared to the non-carrier average of $-0.44 \mathrm{D}(\mathrm{SD}=2.27)$ and the overall cohort average of $-0.45 \mathrm{D}(\mathrm{SD}=2.28)$; the single variant $P$ was 0.000183 . In the IECC, the best potential causal variant was rs62620697, which was predicted damaging by MutationTaster, had a CADD score of 23.8, and had a low single variant p-value of 0.002632 . rs145293758 also had a low p-value (0.000311) but was not predicted damaging. Carriers ( $\mathrm{N}=9)$ of rs62620697 had an average SER of $-2.17 D(S D=6.87)$ compared to that of non-carriers with an average $S E R$ of $0.20(S D=2.27)$. 
Table 1

Potential Missense Causal Variants in Prioritized Genes

\begin{tabular}{|c|c|c|c|c|c|c|c|c|c|}
\hline CHR & BP & rs ID & Gene & AA Change & SIFT & PolyPhen2 & MT & FATHMM & CADD \\
\hline 1 & 7879401 & rs147327372 & PER3 & Thr519Ala & $\mathrm{T}$ & B & $\mathrm{N}$ & $\mathrm{T}$ & 0.01 \\
\hline 1 & 7890153 & rs144178755 & PER3 & Thr1040Asn & $\mathrm{D}$ & B & $\mathrm{N}$ & $\mathrm{T}$ & 0.962 \\
\hline 1 & 216138793 & rs554957414 & USH2A & Pro2329Leu & $\mathrm{D}$ & $\mathrm{D}$ & $\mathrm{D}$ & $\mathrm{D}$ & 29.1 \\
\hline 1 & 216373416 & rs148135241 & USH2A & Ser1122Pro & $\mathrm{D}$ & $D$ & $\mathrm{D}$ & $\mathrm{D}$ & 22.8 \\
\hline 1 & 216419934 & rs201527662 & USH2A & Cys934Trp & $\mathrm{D}$ & $\mathrm{D}$ & $\mathrm{D}$ & D & 36 \\
\hline 3 & 33840234 & rs200697599 & PDCD6IP & Ile5Ser & $\mathrm{D}$ & $\mathrm{D}$ & $\mathrm{D}$ & $\mathrm{T}$ & 32 \\
\hline 3 & 33879764 & rs199990824 & PDCD6IP & Asp376Asn & $\mathrm{D}$ & $B$ & $D$ & $\mathrm{~T}$ & 26 \\
\hline 3 & 33905532 & rs62620697 & PDCD6IP & Ala719Thr & $\mathrm{T}$ & $\mathrm{B}$ & D & $\mathrm{T}$ & 23.8 \\
\hline 3 & 33905587 & rs145293758 & PDCD6IP & Pro737Arg & $\mathrm{T}$ & $\mathrm{B}$ & $\mathrm{N}$ & $\mathrm{T}$ & 20.2 \\
\hline 3 & 49039984 & rs140290144 & P4HTM & Ile227Val & $\mathrm{T}$ & B & $\mathrm{D}$ & $\mathrm{T}$ & 22.1 \\
\hline 3 & 49043292 & rs144279528 & P4HTM & Asp386Asn & $\mathrm{T}$ & B & $\mathrm{D}$ & $\mathrm{T}$ & 27.3 \\
\hline 8 & 102570910 & rs142411476 & GRHL2 & Arg183GIn & $\mathrm{T}$ & $\mathrm{D}$ & $\mathrm{D}$ & $\mathrm{T}$ & 22 \\
\hline 16 & 75512734 & rs140699573 & CHST6 & Gln331His & $\mathrm{D}$ & $\mathrm{D}$ & $\mathrm{D}$ & $\mathrm{D}$ & 27.4 \\
\hline 17 & 44055786 & rs139796158 & MAPT & Ala118Gly & $\mathrm{D}$ & $\mathrm{D}$ & $\mathrm{D}$ & $\mathrm{T}$ & 26.4 \\
\hline 17 & 44060807 & rs76375268 & MAPT & Gly213Arg & $\mathrm{D}$ & $D$ & $\mathrm{~N}$ & $\mathrm{~T}$ & 11.71 \\
\hline 17 & 44060859 & rs63750072 & MAPT & Gln230Arg & $\mathrm{D}$ & $D$ & $\mathrm{D}$ & $\mathrm{T}$ & 4.652 \\
\hline 17 & 44067341 & rs143956882 & MAPT & Ser427Phe & $\mathrm{D}$ & $\mathrm{D}$ & $\mathrm{D}$ & $\mathrm{T}$ & 28.5 \\
\hline 17 & 44101481 & rs63750191 & MAPT & GIn741Lys & $\mathrm{D}$ & $D$ & $\mathrm{D}$ & $\mathrm{T}$ & 27.5 \\
\hline \multicolumn{10}{|c|}{$\begin{array}{l}\text { Legend: The best potential missense causal variants in our top prioritized genes. The headers represent: CHR = } \\
\text { chromosome, } B P=\text { physical position in basepairs (hg19), Gene = gene location, } A A \text { change = amino acid change } \\
\text { caused by mutation, SIFT = pathogenicity prediction from SIFT (where } T=\text { tolerated and } D=\text { damaging), PolyPhen2 } \\
=\text { pathogenicity prediction from PolyPhen2 (where } B=\text { benign and } D=\text { damaging), } M T=\text { pathogenicity prediction } \\
\text { from MutationTaster (where } N=\text { neutral and } D=\text { damaging), FATHMM = pathogenicity prediction from FATHMM } \\
\text { (where } T=\text { tolerated and } D=\text { damaging), CADD = CADD phred score }\end{array}$} \\
\hline
\end{tabular}

Potential candidate variants were also identified in PER3, which was genome-wide significant in REHS and replicated in IECC. The REHS signal was primarily driven by two variants - rs147327372 and rs144178755, which had single variant p-values of $1.72 \times 10^{-8}$ and 0.004953 , respectively. However, neither variant was predicted to be damaging by the prediction algorithms nor appeared in the other European cohorts and were not significant, although rs 147327372 did have a p-value of 0.046 in EPIC-Norfolk.

The signals in the other four genes, identified primarily by the two burden-style tests, were driven by a cumulative effect of several variants. In this case, we relied primarily on annotation and reported variants that were generally agreed upon by multiple prediction programs. Five good candidate variants were located in MAPT: rs139796158, rs76375268, rs63750072, rs143956882, and rs63750191. All these variants were nonsynonymous variants and predicted damaging by three of the four databases (except for rs76375268, which was predicted damaging by two). rs139796158, rs143956882, and rs63750191 all had CADD scores > 26. In CHST6, the best candidate variant was the missense 
variant rs140699573. It was predicted damaging by SIFT, PolyPhen2, MutationTaster, and FATHMM and has a CADD score of 27.4. In GRHL2, the best candidate variant was rs142411476. It was predicted damaging by two databases and had a CADD score of 22. In P4HTM, two variants of interest were identified: rs140290144 and rs144279528. These variants were predicted damaging by MutationTaster and had CADD scores of 22.1 and 27.3, respectively. Finally in USH2A, three variants (rs554957414, rs148135241, and rs201527662) were all predicted damaging by the five prediction algorithms and had CADD scores above 22.

\section{Structural Analysis of Prioritized Candidate Proteins}

In addition to the annotation, we also performed protein structural modeling of all coding variants within the prioritized genes (98 variants across 6 genes/proteins) and calculated free energy difference $(\Delta \Delta \mathrm{G})$ between wildtype and mutant proteins (Supplementary Table 30 ); positive $\Delta \Delta G$ indicates a shift from a more stable to a less stable isoform. More detailed information on the structural analysis can be found in the Supplemental Methods.

In PDCD6IP, both rs145293758 and rs200697599 were predicted to be highly destabilizing to protein structure (Supplementary Fig. 3A). The variant rs145293758 leads to replacement of a proline (Pro737) for an asparagine near phosphorylation sites in the protein's self-associating domain, which could disrupt phosphorylation. rs200697599 (Ile5) and rs199990824 (Asp376) result in changes to the protein's BR01 domain, which is involved in endosomal targeting. The isoleucine to serine mutation at rs200697599 could introduce a phosphorylation site at the N-terminus while the asparagine to aspartic acid mutation at rs199990824 could disrupt hydrogen bonds. Recall that both rs145293758 and rs199990824 were identified as potential causal variants in IECC and EACC, respectively, based on their annotation and single variant $p$-values.

For PER3, several variants may affect structure, including rs140974114, which results a serine (Ser751) to aspartic acid substitution at the protein's nuclear localization signal and could disrupt hydrogen bonds and rs200140283, which results in an alanine (Ala681) to glycine substitution in the CSNK1E binding domain. Further potential disruptions occur at rs139315125 (His416), which takes place in the nuclear export signal 3 and rs77418803 (Ser919), which occurs near the nuclear export signal 2. The model is provided in Supplementary Fig. 3B).

Of the variants in MAPT, two were predicted to be destabilizing (rs76375268 at Gly213 and rs63750191 at GIn741) (Supplementary Fig. 3C). Further, rs73314997 (Ser318) and rs143956882 (Ser427) are located near known pathogenic mutations for frontotemporal dementia and Pick disease of the brain, respectively.

Three variants on the luminal domain of CHST6 were found to have a mild effect on protein stability. Two of these variants (rs201349198 at Ala326 and rs140699573 at GIn331) are positioned near variants known to cause macular corneal dystrophy (MCD) near the C-terminus. This suggests the $\mathrm{C}$-terminus is sensitive to mutations enabling interference with keratan sulfation, which could cause a loss of function that can lead to a milder disease phenotype such as refractive error. The model can be found in Supplementary Fig. 4A.

In GRHL2, variants were only predicted to have a mild effect on protein structure and were not located near known pathogenic variants (Supplementary Fig. 4B).

For P4HTM, rs140290144 is predicted to be moderately destabilizing (Supplementary Fig. 4C). It substitutes a valine for a buried isoleucine (lle227) between two calcium binding sites; potential disruption of these calcium binding sites can result in loss of function. Similarly, rs144279528 occurs in the Fe-dependent 2-OG dioxygenase domain close to an iron binding residue. Substitution of asparagine from the wildtype aspartic acid (Asp386) could have an impact on iron binding by introducing a glycosylation (due to location on protein surface) or disruption of hydrogen bonding. 
Of particular interest in the protein modeling was that of usherin (USH2A), the known retinitis pigmentosa gene. Five variants were predicted to be highly destabilizing, particularly rs 554957414 with a $\Delta \Delta \mathrm{G}$ value of $99.19 \mathrm{kcal} / \mathrm{mol}$ ). Three of these variants, including rs554957414 (Pro2329), result in the loss of proline and the loss of that ring structure could cause an increase in conformational flexibility and account for such high destabilization predictions (Supplementary Fig. 5). Further, a mutation at rs201527662 (Cys934) results in the replacement of cysteine with tryptophan and will disrupt a disulfide bond between two cysteines.

We also compared the $\Delta \Delta \mathrm{G}$ of these five candidate variants with the $\Delta \Delta \mathrm{G}$ of all USH2A ClinVar $(\mathrm{n}=63)$ and gnomAD (n $=1870$ ) variants using the Wilcoxon rank sum test. A significant difference between the ClinVar variants and gnom $A D$ variants was found $(P=0.0008)$ and the $\Delta \Delta G$ values of our candidate variants was much more similar to the known pathogenic variants than the putatively benign GnomAD variants (Supplementary Fig. 6).

\section{Potential Causal Variants in Other Genome-wide Significant Genes}

We also identified variants within the other 122 genome-wide significant genes that had a high potential to be damaging. This included 25 variants across the five cohorts; the results are found in Supplementary Table 31. Like our prioritized genes, we also performed protein modeling on these variants (Supplementary Table 32).

Notable findings from the structural analysis include a valine to phenylalanine substitution (Val105) that would disrupt a helix in ALG3, which has been implicated in congenital disorders of glycosylation that have ocular phenotypes ${ }^{85}$ (Supplementary Fig. 7A). We also identified multiple glycine substitutions in TNFRSF13B in areas associated with heparan sulfate - glycosaminoglycan biosynthesis; heparan sulfate has been shown to play a role in eye pathologies ${ }^{86}$ (Supplementary Fig. 7B).

\section{Discussion}

In this large scale, gene-based analysis of rare variants in refractive error, 129 novel associated genes were identified. Pathway analysis revealed that 59 of these genes were involved in cell cycle, organ morphology, and embryonic development and 21 of these genes had upstream regulators that were directly involved in retinal development or eye morphogenesis. Given the substantial level of missing heritability still present within the refractive error, it is likely that at least some of this heritability is explained by rare variants within these genes. The fact that the significance of these genes and the explained variance of refractive error due to these genes did not significantly change after inclusion of GRS in the analysis, suggests that these association signals are independent from the effects of known common refractive error risk variants.

This is the first large scale meta-analysis using gene-based tests for rare variants in refractive error, which was undertaken to identify rare variants that may be partially responsible for missing heritability, particularly within the CREAM data set ${ }^{21}$. The CREAM data set is well-suited for this type of rare variant analysis. First, we were able to combine many smaller cohorts into two mega-analyses - IECC $(N=11,505)$ and EACC $(N=4,867)$. These metaanalyses greatly boosted power to detect variants with a MAF $\leq 0.01$ and allowed more rare variants to be combined into a single, gene-based marker. In addition, we had three cohorts > 1000 subjects to observe replication and perform the combined meta-analyses. Genes identified in this study were done so across a very large pool of subjects, lowering the potential for type I error.

The multiethnic composition of this dataset also allowed for observation both across and within ethnicities. We have delineated how some genes were found only in Indo-Europeans and other in East Asians, as well as some that cut across the ethnic divide. Thus, we were able to identify risk genes that might be present within a particular population (such as ST6GALNAC5 in IECC) or more universal, like PDCD6IP. 
Among those good candidate genes are PER3, PDCD6IP, MAPT, CHST6, P4HTM, USH2A, and GRHL2. These genes are all known to be associated with ocular abnormalities. PER3 is a circadian rhythm gene; circadian rhythm is associated with refractive error ${ }^{22}$. PDCD6IP and MAPT are both expressed in the retina while CHST6, and GRHL2 are both involved in corneal dystrophy ${ }^{77,87}$. P4HTM affects eye morphology in mice knockouts ${ }^{82}$; it is also notable for being replicated in the UKBB analysis. USH2A is expressed in the retina and is a known RP gene ${ }^{80,81}$.

Five of these prioritized genes were found to be regulated by the cytokine MIF, which has been shown to regulate zebrafish eye development ${ }^{82}$ and have protective effects to photoreceptors ${ }^{84}$. More work on the MIF network with respect to refractive error is needed. We were further able to identify potential causal variants in these prioritized genes and using structural analysis were even able to determine the effect on protein stability.

STON1, C5AR1, and WDFY3 were all replicated in UKBB. C5AR1 is expressed in retinal Müller cells, which are known to play a role in retinal disease ${ }^{88}$. STON1 is associated with $A M D^{89}$ while WDFY3 is associated with inherited retinal dystrophies ${ }^{90}$. Other potential interesting candidates include GDF15, which was a top significant gene across all four meta-analyses, and has been found to be significantly overexpressed in highly myopic eyes ${ }^{91}$ and patients with vitreoretinal disorders ${ }^{92}$ and may also be a potential molecular marker of neurodegeneration in glaucoma ${ }^{93}$, and MRPS27. This gene was genome-wide significant in the meta-analysis and in two individual cohorts, REHS and EPICNorfolk. While MRPS27 is not known to be associated with eye disease, a common variant in this gene was found to be genome-wide significant in the GWAS meta-analysis of refractive error conducted by Hysi et al. ${ }^{22}$. Other candidate genes with known links to eye disease/functions include $H C A R 1$ with glaucoma ${ }^{94,95}$ and $E P B 41 L 2$ with a potential role in phototransduction ${ }^{96}$.

One final interesting set of genes was those that were genome-wide significant within a single cohort. This implies that there may be rare risk variants unique to a certain population that are fixed in other populations. This includes ST6GALNAC5, which was genome-wide significant in IECC in both EMMAX-VT and ACAT $\left(P=5.84 \times 10^{-7}, 9.03 \times 10^{-}\right.$ $\left.{ }^{10}\right)$. This gene catalyzes the transfer of sialic acid; polysialic acid has been shown to prevent vascular damage in retina ${ }^{97}$ and to stimulate the generation of new rods in the retinas of developing zebrafish ${ }^{98}$. Other interesting significant genes unique to a single cohort included SERTAD3 in EACC, which is overexpressed in retinoblastoma ${ }^{99}$ and $K L F 1$ in EPIC-Norfolk, which may be expressed in the eye ${ }^{100}$. We also note that gene-based analyses for refractive error had been previously performed in BDES ${ }^{101}$. Of the five significant genes from that analysis, two were replicated at $\mathrm{P} \leq$ 0.05 - PTCHD2 and CRISP3. PTCHD2 is located near the known myopia locus MYP14 on 1p36.22 ${ }^{69,102}$ and CRISP3 is expressed in the retina ${ }^{101,103}$.

This study used multiple tests (EMMAX-VT, EMMAX-CMC and ACAT) to identify significant genes and looked at overlap to find more robust signals. By using multiple tests that differ slightly in design, we were able to cast a wider net in our search. The ACAT test was particularly useful for identifying potential causal variants within a candidate gene, as it allowed us to observe which variants had significant single variant p-values. This enabled us to zero in on potential causal variants in genes like PDCD6IP and PER3, though we note that highlighting any potential causal variants are speculative at this point. We also felt it prudent to not give more weight to the result of one test over another and instead take the largest number of unique, significant genes since this was a discovery study, though we did try to give more weight to the genes that were identified by all three tests, such as PDCD6IP.

We note that the three tests did not always agree, though the two burden-style tests agreed more often than ACAT. This is not surprising given the different nature of the tests. Both EMMAX-VT and EMMAX-CMC were burden-style tests that create a new, gene-based marker on which the p-value is calculated. The ACAT test was an aggregation-style test

Page 15/30 
created from single variant $p$-values that does not create a new gene-based marker ${ }^{35}$. This is a critical distinction; it means that the markers analyzed in the burden-style tests and the ACAT tests are different. The ACAT analyses may have been slightly underpowered with respect to the burden-style tests, as we used a minimum allele count of three in our analyses. For EMMAX-VT and EMMAX-CMC this was calculated across all variants within a gene and for ACAT at each individual variant, which resulted in certain variants being removed from the ACAT analysis that were present in the burden style analyses. Therefore, genes present in all three analyses indicate a more robust association with refractive error.

Since this is an exome microarray study, there were still large portions of the genome that would not have been covered in this work. Thus, there are almost certainly additional rare risk variants for refractive error; this study simply provides an excellent starting point. These non-genotyped variants could also explain why we did not see replication with

previous refractive error GWAS findings ${ }^{21,22}$. Some of the genes identified in the common variant GWAS may have included rare risk variants that were specific to a particular population that was not used in this study.

Another challenge is that due to the gene-based nature of this work, it is critical to remember that the gene-based markers across the cohorts are often made up of different variants. This means that the gene-based marker for gene $\mathrm{A}$ in IECC might be made up of three variants, and in REHS might be made up of seven variants, two of which are shared across the two cohorts. This means that it was possible that some cohorts may have had association tests that were less significant because of inclusion of non-significant rare variants that did not appear in other cohorts.

We also note that this was an exploratory analysis to determine candidate genes, and one of our goals was to cast a wide net to capture potential candidates. Therefore, we chose a more liberal genome-wide significance threshold, which may allow for potential type I errors but would also ensure that a good candidate gene would not be missed.

This work identified 129 genome-wide significant genes for refractive error using the gene-based rare variant approach. Most of these genes are novel for association with refractive error but many have associations with other ocular abnormalities. This is the largest gene-based study of rare variants performed on refractive error. The fact that we found over 100 significant genes shows that rare variants (MAF $\leq 0.01)$ do account for some of the missing refractive error heritability not identified in the common variant GWAS. We were able to prioritize seven of these genes as our best candidate genes for causality based on biological function - PDCD6IP, MAPT, CHST6, GRHL2, USH2A, P4HTM, and $P E R 3$ as well as GDF15 and MRPS27 based on the strength of association. Validation studies, including replication within additional cohorts, are planned to identify the best candidates for functional studies to unravel the pathophysiology of refractive error and myopia.

\section{Declarations}

\section{Acknowledgments}

The authors gratefully acknowledge Sana Wajid of the Bioinformatics Core of the University of Pennsylvania for her quality control work on these data. This work was funded in part by the Intramural Research Program of the National Human Genome Research Institute, National Institutes of Health. The acknowledgments for each individual study cohort are given alphabetically by study below. APK is supported by a UKRI Future Leaders Fellowship. Molecular graphics and analyses were performed with UCSF ChimeraX, developed by the Resource for Biocomputing, Visualization, and Informatics at the University of California, San Francisco, with support from National Institutes of Health R01-GM129325 and the Office of Cyber Infrastructure and Computational Biology, National Institute of Allergy and Infectious Diseases

Page $16 / 30$ 
AREDS. AREDS was supported by the National Eye Institute (grants R01EY16482, R21EY015145, and P30EY11373) and by Research to Prevent Blindness and the Ohio Lions Eye Research Foundation.

AREDS was also supported by contracts from National Eye Institute/National Institutes of Health, Bethesda, MD, with additional support from Bausch \& Lomb Inc, Rochester, NY. The genotyping costs were supported by the National Eye Institute (R01EY020483 to D.S.) and some of the analyses were supported by the Intramural Research Program of the National Human Genome Research Institute, National Institutes of Health, USA. AREDS acknowledges Frederick Ferris, National Eye Institute, National Institutes of Health, Bethesda, MD; and the Center for Inherited Disease Research, Baltimore, MD where SNP genotyping was carried out. The investigators gratefully acknowledge the advice and guidance of Hemin Chin of the National Eye Institute.

BDES. BDES was supported by the National Eye Institute of the National Institutes of Health under award numbers EY06594 (R. Klein and B. E. K. Klein), EY10605 (B. E. K. Klein) and R01EY021531 (A.P.K and P.D.) and some of the analyses were supported by the Intramural Research Program of the National Human Genome Research Institute, National Institutes of Health, USA.

Croatia-Korcula: The Croatia-Korcula study was funded by the Medical Research Council (UK) "QTL in health and disease" programme core grants, currently MC_UU_00007/10, as well as grants from the Republic of Croatia Ministry of Science, Education and Sports (108-1080315-0302; 216-1080315-0302) and the Croatian Science Foundation (8875). The study acknowledge Dr. Biljana Andrijević Derk, Valentina Lacmanović Lončar, Krešimir Mandić, Antonija Mandić, Ivan Škegro, Jasna Pavičić Astaloš, Ivana Merc, Miljenka Martinović, Petra Kralj, Tamara Knežević and Katja BaraćJuretic as well as the recruitment team from the Croatian Centre for Global Health, University of Split and the Institute of Anthropological Research in Zagreb for the ophthalmological data collection; the Wellcome Trust Clinical facility (Edinburgh, United Kingdom) for Exome array genotyping.

EGCUT: EGCUT was supported by the European Union H2020 grant 692145, Est.RC grant IUT20-60 and the European Regional Development Fund, in the frame of Centre of Excellence in Genomics and Estonian Research Infrastructure's Roadmap and the University of Tartu (SP1GVARENG). This research was supported by NIH grant 5R01 DK07 5787 -13, under subward-agreement GENFD0001B52751; the European Union through Horizon 2020 research and innovation programme under grant 633589 and the European Regional Development Fund (Project No. 2014-2020.4.01.16-0125). This research was also supported by the European Union through the European Regional Development Fund (Project No. 2014-2020.4.01.16-0125) and the Estonian Research Council grant PUT (PRG687) European Union H2020 grant 654248 (Corbel). EGCUT acknowledges the High Performance Computing Center of the University of Tartu.

EPIC-Norfolk. The EPIC-Norfolk study (DOI 10.22025/2019.10.105.00004) has received funding from the Medical Research Council (MR/N003284/1 and MC-UU_12015/1) and Cancer Research UK (C864/A14136). The genetics work in the EPIC-Norfolk study was funded by the Medical Research Council (MC_PC_13048).We are grateful to all the participants who have been part of the project and to the many members of the study teams at the University of Cambridge who have enabled this research.

FITSA: FITSA was supported by ENGAGE (FP7-HEALTH-F4-2007, 201413);

European Union through the GENOMEUTWIN project (QLG2-CT-2002-01254); the Academy of

Finland Center of Excellence in Complex Disease Genetics $(213506,129680)$; the Academy of Finland

Ageing Programme; and the Finnish Ministry of Culture and Education and University of Jyväskylä. FITSA acknowledges the contributions of Emmi Tikkanen, Samuli Ripatti, Markku Kauppinen, Taina Rantanen and Jaakko

Page $17 / 30$ 
Kaprio.

Ogliastra: The Ogliastra Study gratefully acknowledges the population of Ogliastra, Sardinia, Italy. The Ogliastra study was funded by a grant from the Italian Ministry of Education, University and Research (MIUR) $n^{\circ}: 5571 / D S P A R / 2002$.

RSI, ERF: The Rotterdam Study and ERF were supported by European Research Council (ERC) under the European Union's Horizon 2020 research and innovation programme (grant 648268), Netherlands Organisation for Scientific Research (NWO, grant 91815655 to CCWK and NWO Veni 91617076 to VJMV), Ammodo Award (to CCWK), Erasmus Medical Center and Erasmus University, Rotterdam, The Netherlands; Netherlands Organization for Health Research and Development (ZonMw); the Research Institute for Diseases in the Elderly; the Ministry of Education, Culture and Science; the Ministry for Health, Welfare and Sports; the European Commission (DG XII); the Municipality of Rotterdam; the Netherlands Genomics Initiative/NWO; Center for Medical Systems Biology of NGl; Jacoba Breen Fonds, Topcon Europe; Ada Hooghart, Corina Brussee, Riet Bernaerts-Biskop, Amal Hamimida, Patricia van Hilten, Pascal Arp, Jeanette Vergeer, Sander Bervoets. The generation and management of the Illumina exome chip v1.0 array data for the Rotterdam Study (RS-I) was executed by the Human Genotyping Facility of the Genetic Laboratory of the Department of Internal Medicine, Erasmus MC, Rotterdam, The Netherlands. The Exome chip array data set was funded by the Genetic Laboratory of the Department of Internal Medicine, Erasmus MC, from the Netherlands Genomics Initiative (NGI)/Netherlands Organisation for Scientific Research (NWO)-sponsored Netherlands Consortium for Healthy Aging (NCHA; project nr. 050-060-810); the Netherlands Organization for Scientific Research (NWO; project number 184021007) and by the Rainbow Project (RP10; Netherlands Exome Chip Project) of the Biobanking and Biomolecular Research Infrastructure Netherlands (BBMRI-NL; www.bbmri.nl ). We thank Ms. Mila Jhamai, Ms. Sarah Higgins, and Mr. Marijn Verkerk for their help in creating the exome chip database. The authors are grateful to the study participants, the staff from the Rotterdam Study and the participating general practitioners and pharmacists.

REHS. The core management of the Raine Study is funded by the University of Western Australia, Australia; the Telethon Institute for Child Health Research, Australia; Raine Medical Research Foundation, Australia; Women's and Infant's Research Foundation, Australia; Curtin University, Australia; Murdoch University, Australia; Edith Cowan University, Australia; and the University of Notre Dame, Australia. The Generation-2 20-year follow-up of the Raine Study was funded by the National Health and Medical Research Council (NHMRC), Australia: project grant no.: 1021 105. The Generation-2 28-year follow-up of the Raine Study was funded by the NHMRC, Australia: project grants 1121979 and 1 126494

SCES, SiMES, SINDI: The Singapore studies (SCES, SiMES, SINDI) were supported by the National Medical Research Council, Singapore (NMRC 0796/2003, NMRC 1176/2008, STaR/0003/2008; CG/SERI/2010), Biomedical Research Council, Singapore (06/1/21/19/466, 09/1/35/19/616 and 08/1/35/19/550). The Singapore Tissue Network and the Genome Institute of Singapore, Agency for Science, Technology and Research, Singapore provided services.

TwinsUK: TwinsUK received funding from the Wellcome Trust; the European Union MyEuropia Marie Curie Research Training Network; Guide Dogs for the Blind Association; the European 18 Community's FP7 (HEALTHF22008201865GEFOS); ENGAGE (HEALTHF42007201413); the FP-5 GenomEUtwin Project (QLG2CT200201254); US National Institutes of Health/National Eye Institute (1RO1EY018246); NIH Center for Inherited Disease Research; the National Institute for Health Research comprehensive Biomedical Research Centre award to Guy's and St. Thomas' National Health Service Foundation Trust partnering with King's College London. P.G.H. is the recipient of a Fight for Sight ECl award. We acknowledge the contribution of Drs Toby Andrew, Margarida Lopes, Samantha Fahy and Diana Kozareva.

\section{References}

Page $18 / 30$ 
1. Vitale, S., Sperduto, R.D. \& Ferris, F.L., 3rd. Increased prevalence of myopia in the United States between 1971-1972 and 1999-2004. Arch Ophthalmol 127, 1632-9 (2009).

2. Williams, K.M. et al. Increasing Prevalence of Myopia in Europe and the Impact of Education. Ophthalmology 122, 1489-97 (2015).

3. Morgan, I.G., Ohno-Matsui, K. \& Saw, S.M. Myopia. Lancet 379, 1739-48 (2012).

4. Wang, J. et al. Prevalence of myopia and vision impairment in school students in Eastern China. BMC Ophthalmol 20, 2 (2020).

5. Verhoeven, V.J. et al. Visual consequences of refractive errors in the general population. Ophthalmology 122, 1019 (2015).

6. Tideman, J.W. et al. Association of Axial Length With Risk of Uncorrectable Visual Impairment for Europeans With Myopia. JAMA Ophthalmol 134, 1355-1363 (2016).

7. Flitcroft, D.I. The complex interactions of retinal, optical and environmental factors in myopia aetiology. Prog Retin Eye Res 31, 622-60 (2012).

8. Fricke, T.R. et al. Global prevalence of visual impairment associated with myopic macular degeneration and temporal trends from 2000 through 2050: systematic review, meta-analysis and modelling. Br J Ophthalmo/ 102 , 855-862 (2018).

9. Holden, B.A. et al. Global Prevalence of Myopia and High Myopia and Temporal Trends from 2000 through 2050. Ophthalmology 123, 1036-42 (2016).

10. Bourne, R.R. et al. Causes of vision loss worldwide, 1990-2010: a systematic analysis. Lancet Glob Health 1, e33949 (2013).

11. Dolgin, E. The myopia boom. Nature 519, 276-8 (2015).

12. Stambolian, D. Genetic susceptibility and mechanisms for refractive error. Clin Genet 84, 102-8 (2013).

13. Stambolian, D. et al. Meta-analysis of genome-wide association studies in five cohorts reveals common variants in RBFOX1, a regulator of tissue-specific splicing, associated with refractive error. Hum Mol Genet 22, 2754-64 (2013).

14. Fan, Q. et al. Meta-analysis of gene-environment-wide association scans accounting for education level identifies additional loci for refractive error. Nat Commun 7, 11008 (2016).

15. Kiefer, A.K. et al. Genome-wide analysis points to roles for extracellular matrix remodeling, the visual cycle, and neuronal development in myopia. PLoS Genet 9, e1003299 (2013).

16. Shi, Y. et al. Genetic variants at 13q12.12 are associated with high myopia in the Han Chinese population. Am J Hum Genet 88, 805-813 (2011).

17. Nakanishi, H. et al. A genome-wide association analysis identified a novel susceptible locus for pathological myopia at 11q24.1. PLoS Genet 5, e1000660 (2009).

18. Li, Y.J. et al. Genome-wide association studies reveal genetic variants in CTNND2 for high myopia in Singapore Chinese. Ophthalmology 118, 368-75 (2011).

19. Verhoeven, V.J. et al. Genome-wide meta-analyses of multiancestry cohorts identify multiple new susceptibility loci for refractive error and myopia. Nat Genet 45, 314-8 (2013).

20. Verhoeven, V.J. et al. Large scale international replication and meta-analysis study confirms association of the $15 q 14$ locus with myopia. The CREAM consortium. Hum Genet 131, 1467-80 (2012).

21. Tedja, M.S. et al. Genome-wide association meta-analysis highlights light-induced signaling as a driver for refractive error. Nat Genet 50, 834-848 (2018). 
22. Hysi, P.G. et al. Meta-analysis of 542,934 subjects of European ancestry identifies new genes and mechanisms predisposing to refractive error and myopia. Nat Genet 52, 401-407 (2020).

23. Lopes, M.C., Andrew, T., Carbonaro, F., Spector, T.D. \& Hammond, C.J. Estimating heritability and shared environmental effects for refractive error in twin and family studies. Invest Ophthalmol Vis Sci 50, 126-31 (2009).

24. Hysi, P.G., Wojciechowski, R., Rahi, J.S. \& Hammond, C.J. Genome-wide association studies of refractive error and myopia, lessons learned, and implications for the future. Invest Ophthalmol Vis Sci 55, 3344-51 (2014).

25. Pärssinen, O., Kauppinen, M., Kaprio, J., Koskenvuo, M. \& Rantanen, T. Heritability of refractive astigmatism: a population-based twin study among 63- to 75-year-old female twins. Invest Ophthalmol Vis Sci 54, 6063-7 (2013).

26. Pärssinen, O. et al. Heritability of spherical equivalent: a population-based twin study among 63- to 76-year-old female twins. Ophthalmology 117, 1908-11 (2010).

27. Manolio, T.A. et al. Finding the missing heritability of complex diseases. Nature 461, 747 - 53 (2009).

28. Li, B. \& Leal, S.M. Methods for detecting associations with rare variants for common diseases: application to analysis of sequence data. Am J Hum Genet 83, 311-21 (2008).

29. Price, A.L. et al. Pooled association tests for rare variants in exon-resequencing studies. Am J Hum Genet 86, 8328 (2010).

30. Purcell, S. et al. PLINK: a tool set for whole-genome association and population-based linkage analyses. Am J Hum Genet 81, 559-75 (2007).

31. Conomos, M.P., Miller, M.B. \& Thornton, T.A. Robust inference of population structure for ancestry prediction and correction of stratification in the presence of relatedness. Genet Epidemio/39, 276-93 (2015).

32. Kang, H.M. et al. Variance component model to account for sample structure in genome-wide association studies. Nat Genet 42, 348-54 (2010).

33. Price, A.L., Zaitlen, N.A., Reich, D. \& Patterson, N. New approaches to population stratification in genome-wide association studies. Nat Rev Genet 11, 459-63 (2010).

34. Moutsianas, L. et al. The power of gene-based rare variant methods to detect disease-associated variation and test hypotheses about complex disease. PLoS Genet 11, e1005165 (2015).

35. Liu, Y. et al. ACAT: A Fast and Powerful p Value Combination Method for Rare-Variant Analysis in Sequencing Studies. Am J Hum Genet 104, 410-421 (2019).

36. Fisher, R.A. Statistical methods for research workers, (Oliver and Boyd, Edinburgh, 1925).

37. Dewey, M. metap: meta-analysis of significance values. R package version 1.4 edn (2020).

38. Krämer, A., Green, J., Pollard, J., Jr. \& Tugendreich, S. Causal analysis approaches in Ingenuity Pathway Analysis. Bioinformatics 30, 523-30 (2014).

39. Watanabe, K., Umićević Mirkov, M., de Leeuw, C.A., van den Heuvel, M.P. \& Posthuma, D. Genetic mapping of cell type specificity for complex traits. Nat Commun 10, 3222 (2019).

40. Watanabe, K., Taskesen, E., van Bochoven, A. \& Posthuma, D. Functional mapping and annotation of genetic associations with FUMA. Nat Commun 8, 1826 (2017).

41. Fritsche, L.G. et al. A large genome-wide association study of age-related macular degeneration highlights contributions of rare and common variants. Nat Genet 48, 134-43 (2016).

42. Cowan, C.S. et al. Cell Types of the Human Retina and Its Organoids at Single-Cell Resolution. Cell 182, 1623-1640 e34 (2020).

43. Bauer-Mehren, A., Rautschka, M., Sanz, F. \& Furlong, L.I. DisGeNET: a Cytoscape plugin to visualize, integrate, search and analyze gene-disease networks. Bioinformatics 26, 2924-6 (2010). 
44. Günther, S. et al. SuperTarget and Matador: resources for exploring drug-target relationships. Nucleic Acids Res 36, D919-22 (2008).

45. Whirl-Carrillo, M. et al. Pharmacogenomics knowledge for personalized medicine. Clin Pharmacol Ther 92, 414-7 (2012).

46. Szklarczyk, D. et al. STITCH 5: augmenting protein-chemical interaction networks with tissue and affinity data. Nucleic Acids Res 44, D380-4 (2016).

47. Wishart, D.S. et al. DrugBank 5.0: a major update to the DrugBank database for 2018. Nucleic Acids Res 46, D1074D1082 (2018).

48. Wang, K., Li, M. \& Hakonarson, H. ANNOVAR: functional annotation of genetic variants from high-throughput sequencing data. Nucleic Acids Res 38, e164 (2010).

49. Yang, H. \& Wang, K. Genomic variant annotation and prioritization with ANNOVAR and wANNOVAR. Nat Protoc 10, 1556-66 (2015).

50. Chang, X. \& Wang, K. wANNOVAR: annotating genetic variants for personal genomes via the web. J Med Genet 49, 433-6 (2012).

51. Sim, N.L. et al. SIFT web server: predicting effects of amino acid substitutions on proteins. Nucleic Acids Res 40, W452-7 (2012).

52. Adzhubei, I., Jordan, D.M. \& Sunyaev, S.R. Predicting functional effect of human missense mutations using PolyPhen-2. Curr Protoc Hum Genet Chap. 7, Unit7 20 (2013).

53. Schwarz, J.M., Cooper, D.N., Schuelke, M. \& Seelow, D. MutationTaster2: mutation prediction for the deepsequencing age. Nat Methods 11, 361-2 (2014).

54. Rentzsch, P., Witten, D., Cooper, G.M., Shendure, J. \& Kircher, M. CADD: predicting the deleteriousness of variants throughout the human genome. Nucleic Acids Res 47, D886-D894 (2019).

55. Shihab, H.A. et al. Predicting the functional, molecular, and phenotypic consequences of amino acid substitutions using hidden Markov models. Hum Mutat 34, 57-65 (2013).

56. Berman, H.M. et al. The Protein Data Bank. Nucleic Acids Res 28, 235 - 42 (2000).

57. Schymkowitz, J. et al. The FoldX web server: an online force field. Nucleic Acids Res 33, W382-8 (2005).

58. Pettersen, E.F. et al. UCSF ChimeraX: Structure visualization for researchers, educators, and developers. Protein Sci 30, 70-82 (2021).

59. Khanna, T., Hanna, G., Sternberg, M.J.E. \& David, A. Missense3D-DB web catalogue: an atom-based analysis and repository of $4 \mathrm{M}$ human protein-coding genetic variants. Hum Genet 140, 805-812 (2021).

60. Paschalis, E.I. et al. Microglia Regulate Neuroglia Remodeling in Various Ocular and Retinal Injuries. J Immunol 202, 539-549 (2019).

61. Wolf, A., Aslanidis, A. \& Langmann, T. Retinal expression and localization of Mef2c support its important role in photoreceptor gene expression. Biochem Biophys Res Commun 483, 346-351 (2017).

62. Sun, J., Yoon, J., Lee, M., Hwang, Y.S. \& Daar, I.O. Sprouty2 regulates positioning of retinal progenitors through suppressing the Ras/Raf/MAPK pathway. Sci Rep 10, 13752 (2020).

63. Wei, J., Jiang, H., Gao, H. \& Wang, G. Raf-1 Kinase Inhibitory Protein (RKIP) Promotes Retinal Ganglion Cell Survival and Axonal Regeneration Following Optic Nerve Crush. J Mol Neurosci 57, 243-8 (2015).

64. Sowden, J.C., Holt, J.K., Meins, M., Smith, H.K. \& Bhattacharya, S.S. Expression of Drosophila omb-related T-box genes in the developing human and mouse neural retina. Invest Ophthalmol Vis Sci 42, 3095-102 (2001).

65. Koshiba-Takeuchi, K. et al. Tbx5 and the retinotectum projection. Science $287,134-7$ (2000). 
66. Oda, M., Yamamoto, H., Matsumoto, H., Ishizaki, Y. \& Shibasaki, K. TRPC5 regulates axonal outgrowth in developing retinal ganglion cells. Lab Invest 100, 297-310 (2020).

67. Simpson, C.L. et al. Exome genotyping and linkage analysis identifies two novel linked regions and replicates two others for myopia in Ashkenazi Jewish families. BMC Med Genet 20, 27 (2019).

68. Musolf, A.M. et al. Genome-wide scans of myopia in Pennsylvania Amish families reveal significant linkage to 12q15, 8q21.3 and 5p15.33. Hum Genet 138, 339-354 (2019).

69. Wojciechowski, R. et al. Genomewide scan in Ashkenazi Jewish families demonstrates evidence of linkage of ocular refraction to a QTL on chromosome 1p36. Hum Genet 119, 389-99 (2006).

70. Archer, S.N., Schmidt, C., Vandewalle, G. \& Dijk, D.J. Phenotyping of PER3 variants reveals widespread effects on circadian preference, sleep regulation, and health. Sleep Med Rev 40, 109-126 (2018).

71. Stone, R.A. et al. Image defocus and altered retinal gene expression in chick: clues to the pathogenesis of ametropia. Invest Ophthalmol Vis Sci 52, 5765-77 (2011).

72. Stone, R.A. et al. Visual Image Quality Impacts Circadian Rhythm-Related Gene Expression in Retina and in Choroid: A Potential Mechanism for Ametropias. Invest Ophthalmol Vis Sci 61, 13 (2020).

73. Subramanian, L. et al. Ca2 + binding to EF hands 1 and 3 is essential for the interaction of apoptosis-linked gene-2 with Alix/AIP1 in ocular melanoma. Biochemistry 43, 11175-86 (2004).

74. Zhou, H.J. et al. AIP1 mediates vascular endothelial cell growth factor receptor-3-dependent angiogenic and lymphangiogenic responses. Arterioscler Thromb Vasc Bio/ 34, 603-15 (2014).

75. Gupta, N., Fong, J., Ang, L.C. \& Yücel, Y.H. Retinal tau pathology in human glaucomas. Can J Ophthalmo/ 43, 53-60 (2008).

76. Nakazawa, K. et al. Defective processing of keratan sulfate in macular corneal dystrophy. J Biol Chem 259, 137517 (1984).

77. Liskova, P. et al. Ectopic GRHL2 Expression Due to Non-coding Mutations Promotes Cell State Transition and Causes Posterior Polymorphous Corneal Dystrophy 4. Am J Hum Genet 102, 447-459 (2018).

78. Rahikkala, E. et al. Biallelic loss-of-function P4HTM gene variants cause hypotonia, hypoventilation, intellectual disability, dysautonomia, epilepsy, and eye abnormalities (HIDEA syndrome). Genet Med 21, 2355-2363 (2019).

79. Leinonen, $\mathrm{H}$. et al. Lack of P4H-TM in mice results in age-related retinal and renal alterations. Hum Mol Genet 25, 3810-3823 (2016).

80. McGee, T.L., Seyedahmadi, B.J., Sweeney, M.O., Dryja, T.P. \& Berson, E.L. Novel mutations in the long isoform of the USH2A gene in patients with Usher syndrome type II or non-syndromic retinitis pigmentosa. J Med Genet 47, 499506 (2010).

81. Fu, J. et al. Novel compound heterozygous nonsense variants, p.L150* and p.Y3565*, of the USH2A gene in a Chinese pedigree are associated with Usher syndrome type IIA. Mol Med Rep 22, 3464-3472 (2020).

82. Ito, K., Yoshiura, Y., Ototake, M. \& Nakanishi, T. Macrophage migration inhibitory factor (MIF) is essential for development of zebrafish, Danio rerio. Dev Comp Immunol 32, 664-72 (2008).

83. Abu El-Asrar, A.M. et al. The Proinflammatory and Proangiogenic Macrophage Migration Inhibitory Factor Is a Potential Regulator in Proliferative Diabetic Retinopathy. Front Immunol 10, 2752 (2019).

84. Kim, B. et al. MIF Inhibitor ISO-1 Protects Photoreceptors and Reduces Gliosis in Experimental Retinal Detachment. Sci Rep 7, 14336 (2017).

85. Morava, E. et al. Ophthalmological abnormalities in children with congenital disorders of glycosylation type I. Br J Ophthalmol 93, 350-4 (2009).

86. Park, P.J. \& Shukla, D. Role of heparan sulfate in ocular diseases. Exp Eye Res 110, 1-9 (2013).

Page 22/30 
87. Aldave, A.J. et al. Novel mutations in the carbohydrate sulfotransferase gene (CHST6) in American patients with macular corneal dystrophy. Am J Ophthalmol 137, 465-73 (2004).

88. Cheng, L. et al. Modulation of retinal Müller cells by complement receptor C5aR. Invest Ophthalmol Vis Sci 54, 8191-8 (2013).

89. Kawashima-Kumagai, K. et al. A genome-wide association study identified a novel genetic loci STON1GTF2A1L/LHCGR/FSHR for bilaterality of neovascular age-related macular degeneration. Sci Rep 7, 7173 (2017).

90. Martín-Sánchez, M. et al. A Multi-Strategy Sequencing Workflow in Inherited Retinal Dystrophies: Routine Diagnosis, Addressing Unsolved Cases and Candidate Genes Identification. Int J Mol Sci 21(2020).

91. Zhu, X. et al. Profiling and Bioinformatic Analysis of Differentially Expressed Cytokines in Aqueous Humor of High Myopic Eyes - Clues for Anti-VEGF Injections. Curr Eye Res 45, 97-103 (2020).

92. Ilhan, H.D., Bilgin, A.B., Toylu, A., Dogan, M.E. \& Apaydin, K.C. The Expression of GDF-15 in the Human Vitreous in the Presence of Retinal Pathologies with an Inflammatory Component. Ocul Immunol Inflamm 24, 178-83 (2016).

93. Ban, N., Siegfried, C.J. \& Apte, R.S. Monitoring Neurodegeneration in Glaucoma: Therapeutic Implications. Trends Mol Med 24, 7-17 (2018).

94. Kolko, M. et al. Lactate Transport and Receptor Actions in Retina: Potential Roles in Retinal Function and Disease. Neurochem Res 41, 1229-36 (2016).

95. Harun-Or-Rashid, M. \& Inman, D.M. Reduced AMPK activation and increased HCAR activation drive antiinflammatory response and neuroprotection in glaucoma. J Neuroinflammation 15, 313 (2018).

96. Cheng, C.L. \& Molday, R.S. Interaction of 4.1G and cGMP-gated channels in rod photoreceptor outer segments. J Cell Sci 126, 5725-34 (2013).

97. Karlstetter, M. et al. Polysialic acid blocks mononuclear phagocyte reactivity, inhibits complement activation, and protects from vascular damage in the retina. EMBO Mol Med 9, 154-166 (2017).

98. Kustermann, S., Hildebrandt, H., Bolz, S., Dengler, K. \& Kohler, K. Genesis of rods in the zebrafish retina occurs in a microenvironment provided by polysialic acid-expressing Muller glia. J Comp Neuro/ 518, 636-46 (2010).

99. Jansen, R.W. et al. MR Imaging Features of Retinoblastoma: Association with Gene Expression Profiles. Radiology 288, 506-515 (2018).

100. Chiambaretta, F. et al. Cell and tissue specific expression of human Kruppel-like transcription factors in human ocular surface. Mol Vis 10, 901-9 (2004).

101. Chen, F. et al. Variation in PTCHD2, CRISP3, NAP1L4, FSCB, and AP3B2 associated with spherical equivalent. Mol Vis 22, 783-96 (2016).

102. Li, Y.J. et al. An international collaborative family-based whole-genome linkage scan for high-grade myopia. Invest Ophthalmol Vis Sci 50, 3116-27 (2009).

103. Wu, C. et al. BioGPS: an extensible and customizable portal for querying and organizing gene annotation resources. Genome Biol 10, R130 (2009).

\section{Figures}



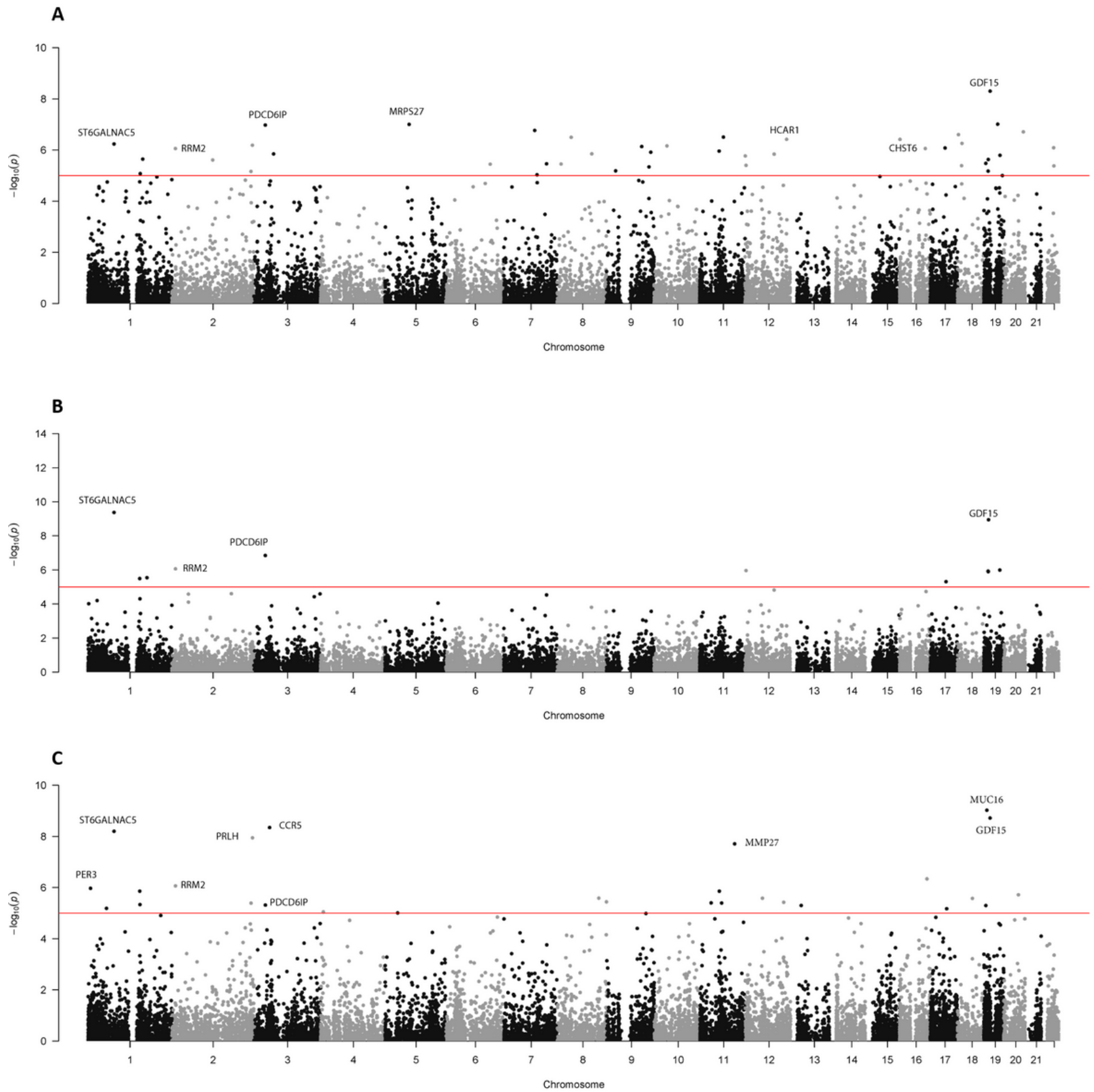

Figure 1

P-values of the multiethnic meta-analysis. The p-values of the meta-analysis combining all five cohorts using the $A$ ) EMMAX-VT test, B) EMMAX-CMC test, and C) ACAT. The line represents the genome-wide significant threshold of $1 \times 10$ 5 . 


\section{EMMAX-VT}

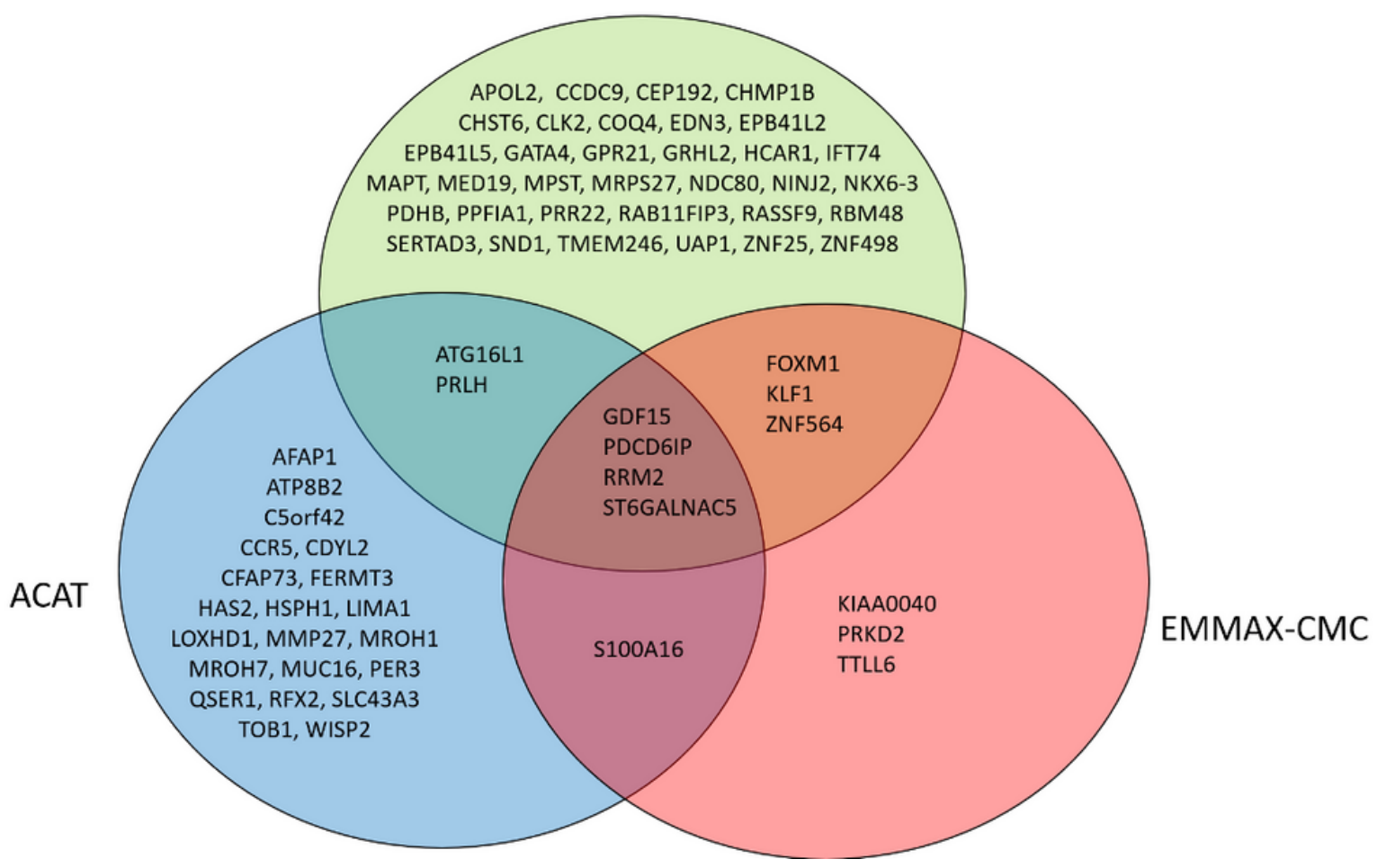

\section{Figure 2}

Overlap between three tests in the multiethnic meta-analysis. A Venn diagram showing the overlap and unique genes in the multiethnic meta-analysis using the three different tests: EMMAX-VT (green), EMMAX-CMC (red), and ACAT (blue). 

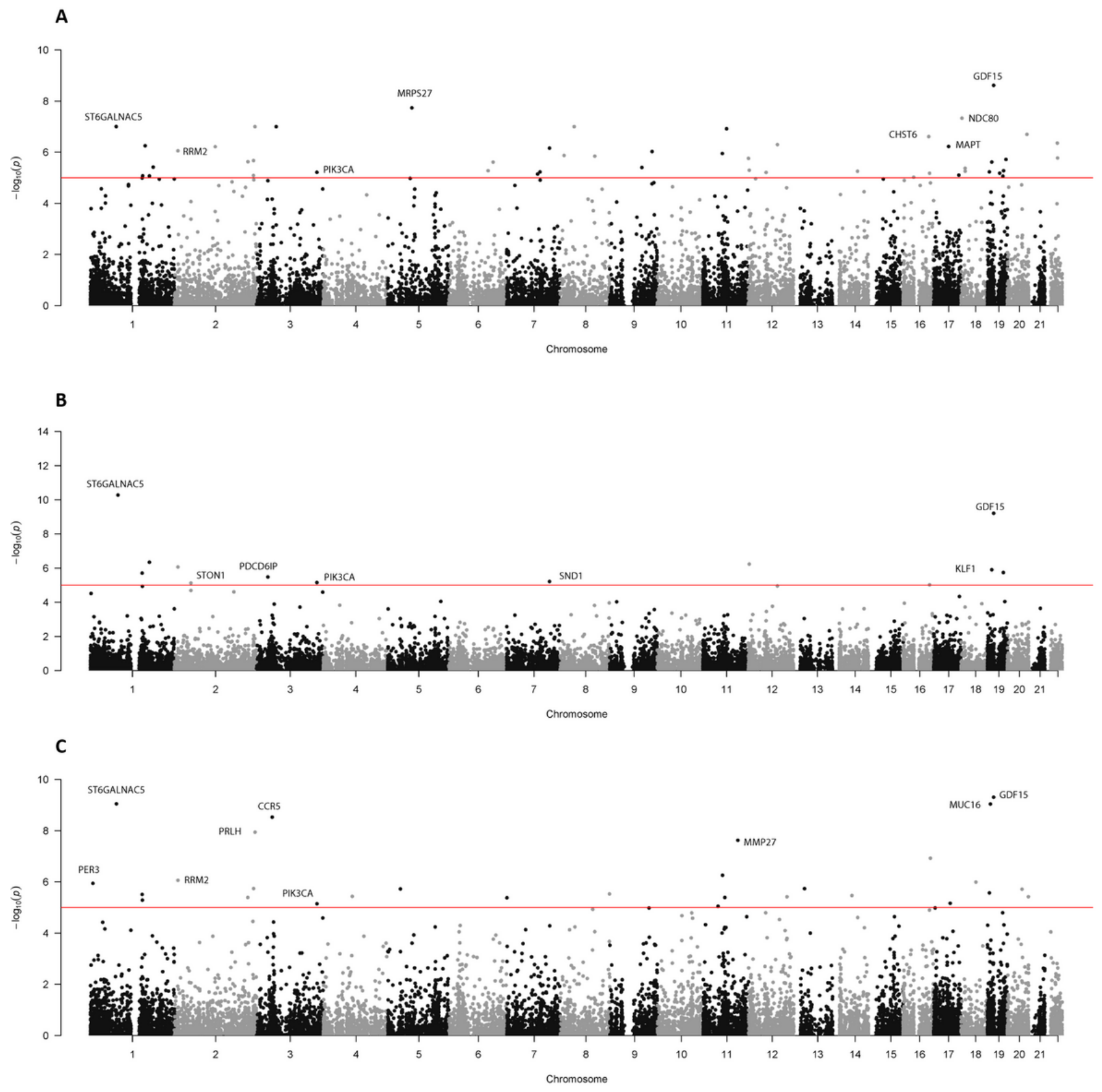

Figure 3

P-values of the Indo-European meta-analysis. The p-values of the meta-analysis combining the four Indo-European derived cohorts using the A) EMMAX-VT test, B) EMMAX-CMC test, and C) ACAT. The line represents the genome-wide significant threshold of $1 \times 10-5$. 


\section{EMMAX-VT}

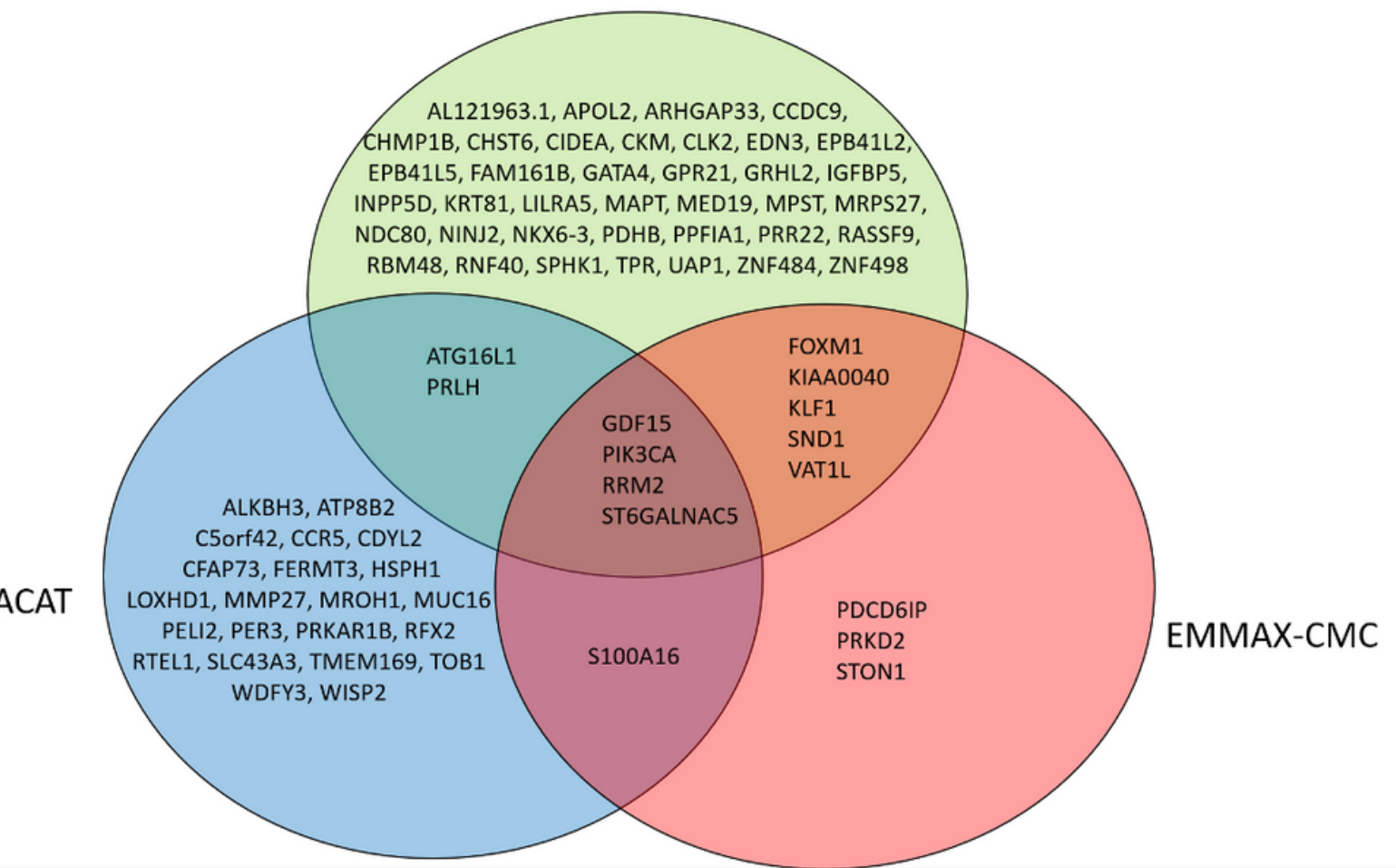

\section{Figure 4}

Overlap between three tests in the Indo-European meta-analysis. A Venn diagram showing the overlap and unique genes in the Indo-European cohorts meta-analysis using the three different tests: EMMAX-VT (green), EMMAX-CMC (red), and ACAT (blue). 

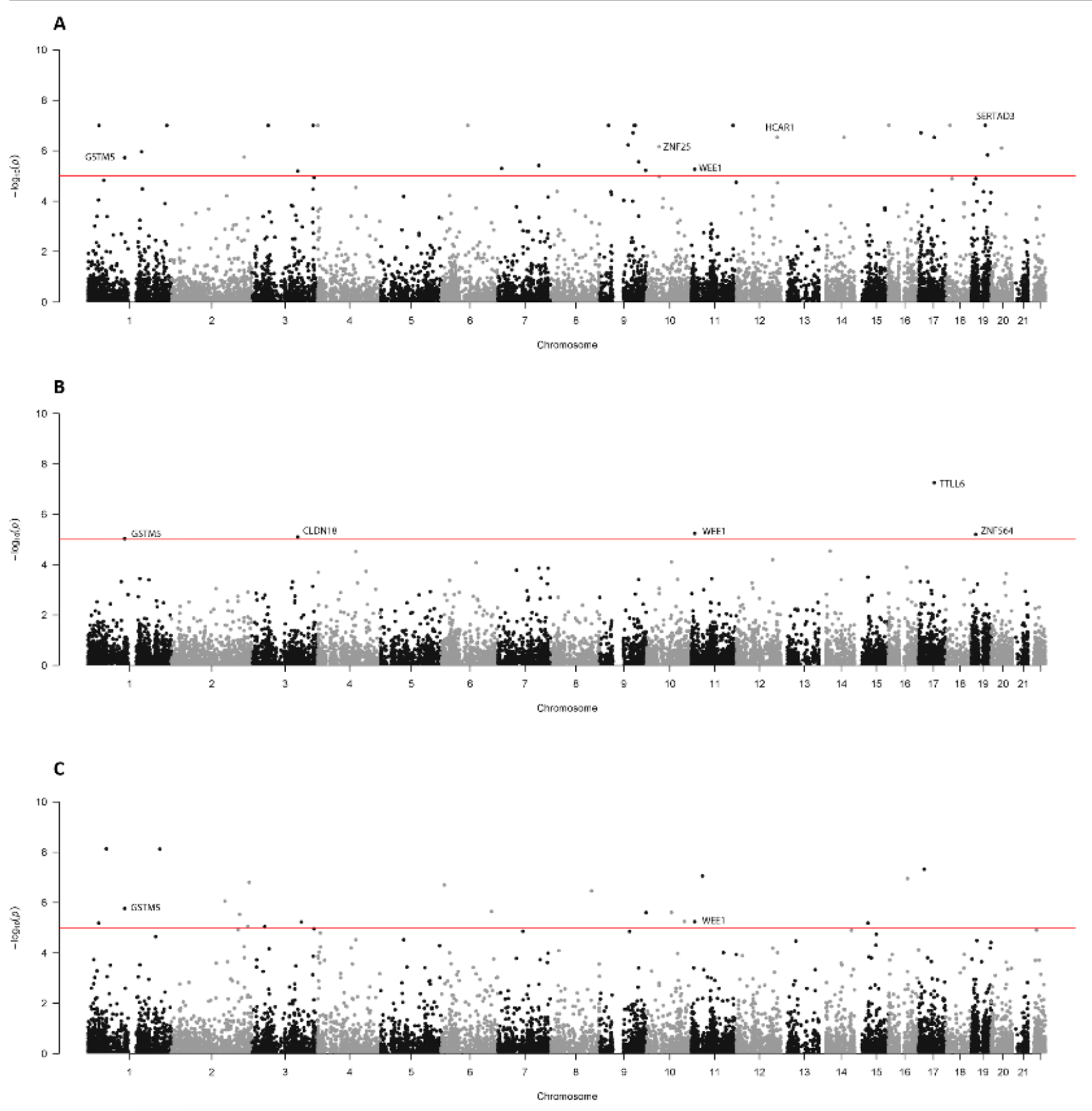

Figure 5

P-values of the analysis using the EACC only. The p-values of the EACC analysis using the A) EMMAX-VT test, B) EMMAX-CMC test, and C) ACAT. The line represents the genome-wide significant threshold of $1 \times 10-5$. 


\section{EMMAX-VT}

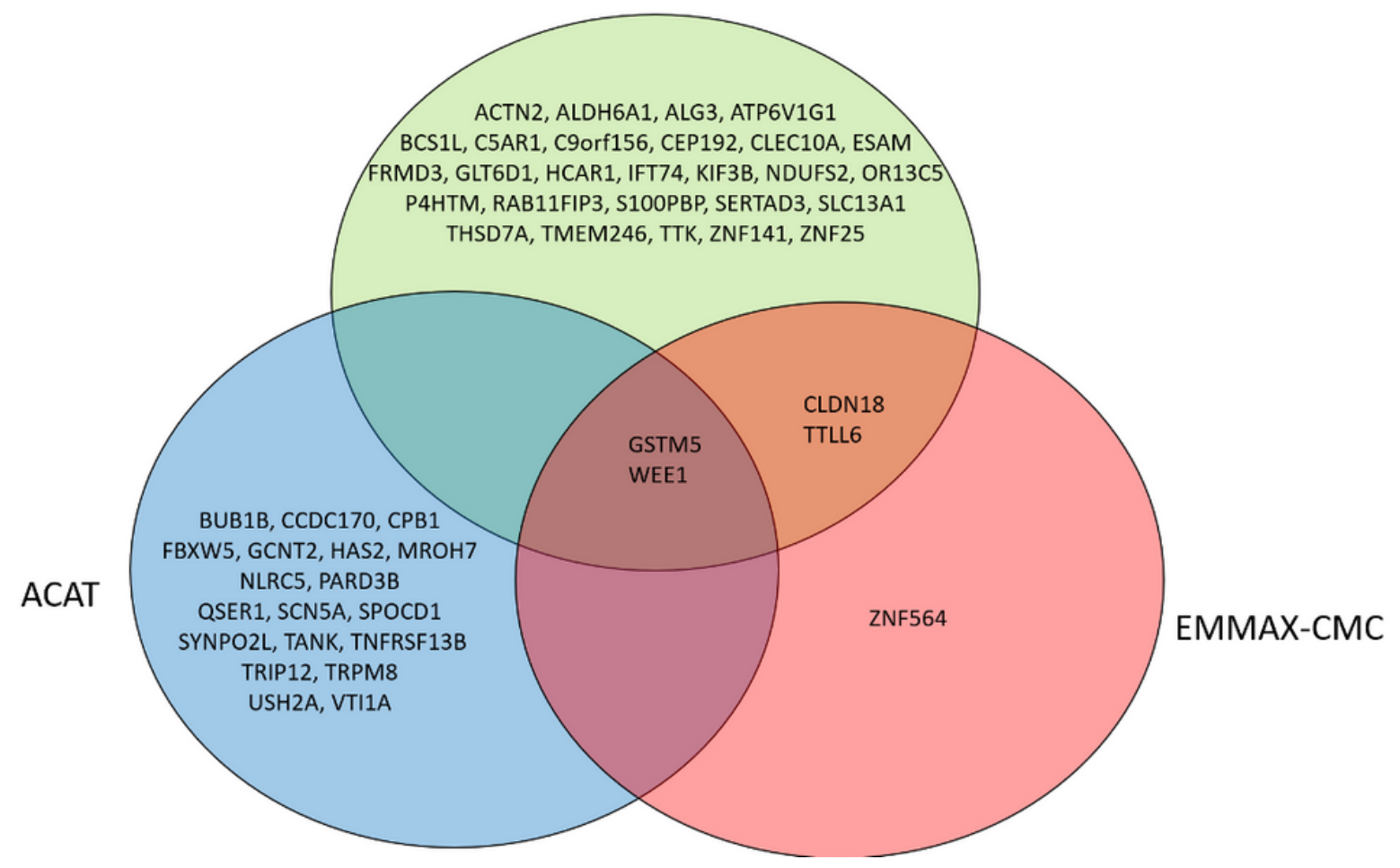

\section{Figure 6}

Overlap between three tests in the EACC analysis. A Venn diagram showing the overlap and unique genes in the EACC analysis using the three different tests: EMMAX-VT (green), EMMAX-CMC (red), and ACAT (blue).

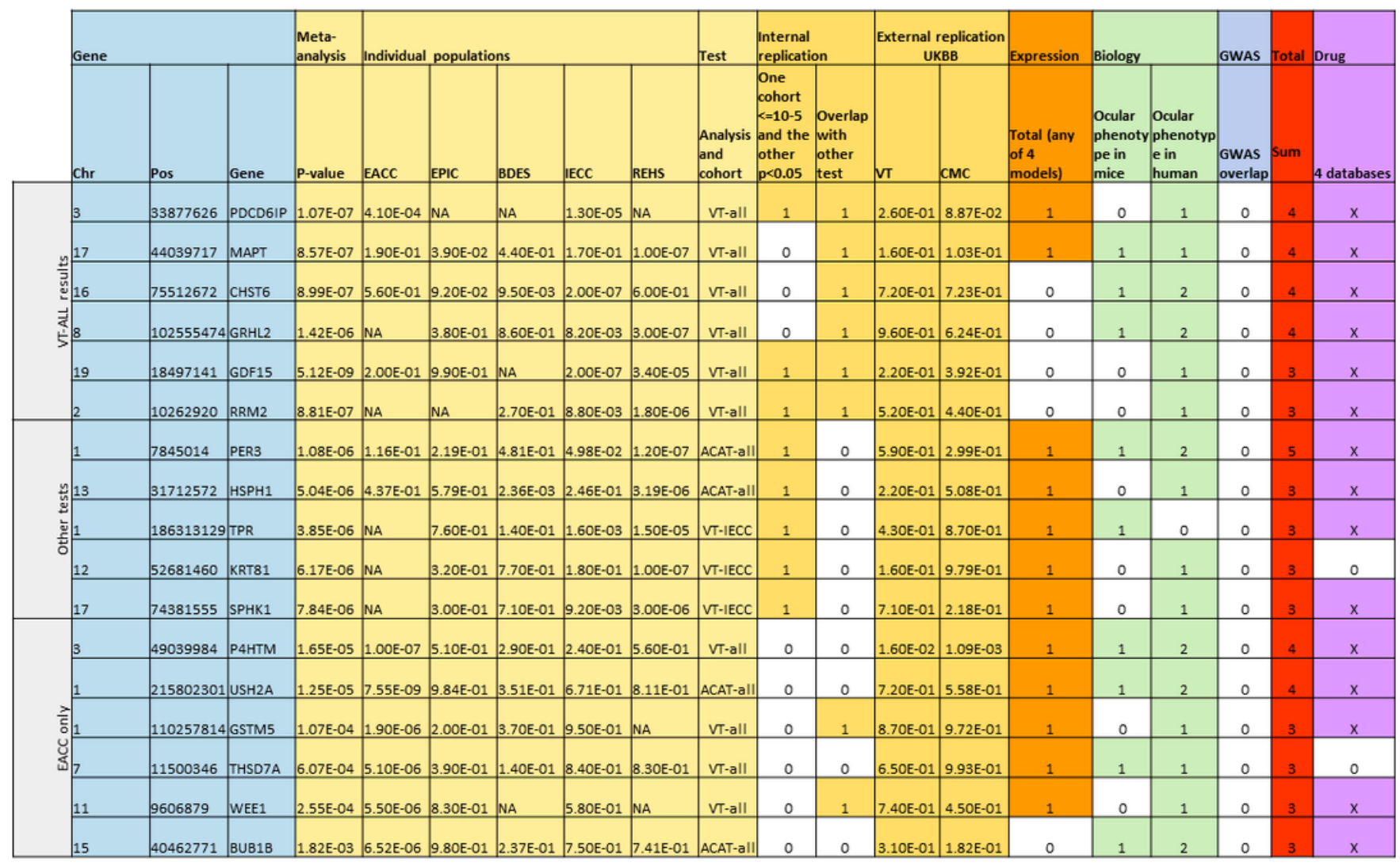

Figure 7 
Prioritization of top genes from all 129 genome-wide significant genes. The top genes ranked by our prioritization schema. The figure contains the chromosome, basepair position, gene name, as well as the meta-analysis $\mathrm{p}$-value and the individual cohort $p$-values for each gene. It also contains which test the given meta-analysis p-value was significant, how many times the gene replicated in our internal analyses. Finally, it contains information regarding gene expression, whether the gene has a known ocular phenotype in mice or humans, overlap with the GWAS performed by Hysi et al., and the final overall prioritization score.

\section{Supplementary Files}

This is a list of supplementary files associated with this preprint. Click to download.

- SupplementaryMethods.docx

- CREAMauthorsandaffiliations.docx

- SuppFig1.tif

- SuppFig2.tif

- SuppFig3.tif

- SuppFig4.tif

- SuppFig5.tif

- SuppFig6.tif

- SuppFig7.tif

- Supplementarytables112final.xlsx

- Supplementarytables1325final.xIsx

- Supplementarytable26.xlsx

- Supplementarytables2732.xlsx 